\title{
An Objective Method for Determining Ocean Mixed Layer Depth with Applications to WOCE Data ${ }^{\mathscr{O}}$
}

\author{
PENG-Qi HuANG \\ State Key Laboratory of Tropical Oceanography, South China Sea Institute of Oceanology, Chinese Academy of Sciences, \\ Guangzhou, and University of Chinese Academy of Sciences, Beijing, China \\ YUAN-ZHENG LU AND SHENG-QI ZHOU \\ State Key Laboratory of Tropical Oceanography, South China Sea Institute of Oceanology, Chinese Academy of Sciences, \\ Guangzhou, China
}

(Manuscript received 6 June 2017, in final form 2 October 2017)

\begin{abstract}
A new method is developed to identify the mixed layer depth (MLD) from individual temperature or density profiles. A relative variance profile is obtained that is the ratio between the standard deviation and the maximum variation of the temperature (density) from the sea surface, and the depth of the minimum relative variance is defined as the MLD. The new method is robust in finding the MLD under the influence of random noise (noise level $\leq 5 \%$ ). A comparison with other available methods, which include the threshold (difference, difference interpolation, gradient, and hybrid methods) and objective (curvature and maximum angle methods) methods, is carried out using the World Ocean Circulation Experiment (WOCE) data. It is found that for a variety of depth sampling resolutions ranging from 0.04 to $25 \mathrm{dbar}$, the new method and the difference-interpolation method predict MLD values that are closer to the visually inspected ones than those by other methods. Moreover, the quality index (QI) of the MLD that is determined by the new method is the highest when compared with those of the available methods. Also, the application of the new method on the WOCE global dataset yields $94 \%$ of MLD values with QI $>0.5$, substantially higher than those $(\leq 86 \%)$ of other methods. Ultimately, it is found that the new method determines very similar MLD values when applied to temperature or density profiles globally because it identifies the base of the mixed layer rather than the uppermost depth of the thermocline. This unique advantage makes the new method applicable in many cases, especially when the density profile is unavailable.
\end{abstract}

\section{Introduction}

The upper-ocean mixed layer (ML) is generally characterized by a vertically homogeneous profile of temperature, salinity, and density. This layer results from vertical mixing near the surface is promoted by various processes-wind stirring, waves, and turbulence-generated by vertical shear or nighttime convective mixing. Hence, the ML is important in establishing the mean state and variability of the World Ocean because it acts as a buffer between the atmosphere and the interior ocean for

Supplemental information related to this paper is available at the Journals Online website: https://doi.org/10.1175/JTECH-D-170104.s1.

Corresponding author: Sheng-Qi Zhou, sqzhou@scsio.ac.cn exchanging heat, momentum, freshwater, and gases (Kantha and Clayson 1999; Wunsch and Ferrari 2004; Vallis 2006). Because of the increasing attention upon climate change or global warming, the ML has been extensively studied by both oceanographers and meteorologists (e.g., Deser et al. 1996; Hanawa and Talley 2001; Yu and Weller 2007; Oka et al. 2015). The ML can be basically characterized by its thickness, typically called mixed layer depth (MLD). The MLD influences the evolution of sea surface temperature (e.g., Carton et al. 2008) and modulates the heat content of the ML (e.g., Godfrey and Lindstrom 1989; Dong et al. 2008), which is highly related to a wide variety of phenomena, from tropical cyclone formation and phytoplankton bloom critical depth theory to climate variability. Therefore, accurate determination of the MLD is a priority in studying these important physical and biology processes in the upper ocean. 
A significant amount of work has been undertaken in developing methods of reliable calculation of the MLD (Defant 1936; Wyrtki 1964; Levitus 1982; Lukas and Lindstrom 1991; Brainerd and Gregg 1995; Obata et al. 1996; Kara et al. 2000; Lavender et al. 2002; Thomson and Fine 2003; Lorbacher et al. 2006; Holte and Talley 2009; Chu and Fan 2011). Temperature, salinity, and density are approximately homogeneous within the ML, and they present sharp gradients beneath the ML. The aforementioned studies are aimed at automatically specifying the lower bound of the ML (i.e., its intersection with the underlying water column) by implementing some criteria on discretized temperature or density profiles. Among these methods, the ones based on a threshold value from a reference depth of $10 \mathrm{~m}$ have been most widespread because they are simple and can presumably be applied to profiles with various depth resolutions (Ohno et al. 2004). These threshold methods are based on the changes in temperature and density [see Kara et al. (2000), Thomson and Fine (2003), de Boyer Montégut et al. (2004), Lorbacher et al. (2006), and Holte and Talley (2009) for summaries of various thresholds] or in their gradients (Lukas and Lindstrom 1991). These threshold methods have been largely employed to evaluate the ML of single profiles and to generate the climatological field (Kara et al. 2003; de Boyer Montégut et al. 2004; Holte and Talley 2009). Generally speaking, the depth at which the local density (temperature) starts differing from the surface density (temperature) by $0.01-0.03 \mathrm{~kg} \mathrm{~m}^{-3}\left(0.01^{\circ}-1^{\circ} \mathrm{C}\right)$ is considered as an MLD proxy. There are also many other relatively sophisticated definitions, such as using a hybrid method based on the results from the threshold methods (Holte and Talley 2009), a step-function least squares regression method and integral depth-scale method used for coarse-resolution profiles (Freeland et al. 1997; Thomson and Fine 2003), a split-merge method based on profile shape (Pavlidis and Horowitz 1974; Thomson and Fine 2003) or dissolved oxygen profiles (Castro-Morales and Kaiser 2012), a curvaturebased method (Lorbacher et al. 2006), and a linear optimal fitting method (further developed as a maximum angle method) (Chu and Fan 2010, 2011).

Depending critically upon the choice of threshold value, the threshold methods are somewhat subjective as a result of the selection of fixed threshold values. As was tested by Brainerd and Gregg (1995), Kara et al. (2000, 2003), de Boyer Montégut et al. (2004), Lorbacher et al. (2006), Holte and Talley (2009), and Ohno et al. (2009) for profiles of temperature or density, a threshold chosen subjectively for one region or one season might not be applicable to another region or season. For example, Ohno et al. (2009) found that the optimal threshold varies from $0.06 \mathrm{~kg} \mathrm{~m}^{-3}$ in the tropics and subtropics to $0.09 \mathrm{~kg} \mathrm{~m}^{-3}$ in the eastern subarctic. Moreover, as was pointed out by de Boyer Montégut et al. (2004) and Lorbacher et al. (2006), the shortcomings of the threshold methods are obvious, especially regarding profiles with gradual pycnoclines. Typically, a smaller threshold can lead to a shallower MLD, and the threshold methods often miss the MLD when a small jump occurs in the ML. Therefore, it is difficult to determine a universal threshold value for all ocean profiles.

In order to objectively determine the MLD, a fewand less dependent on fixed criteria- complicated methods have been developed. The split-merge method proposed by Thomson and Fine (2003) is based on the optimal analysis of linear segments of a density profile. They found that their method performed similarly to the threshold methods, but with a slight improvement in depth determination. The curvature method, as tested by Chu and Fan (2011), is less valid when identifying the MLD from the glider data. As the curvature method deals with the second derivative, it was reported that this method often produced the problematic MLD with the "wriggly" profiles (Lorbacher et al. 2006; Chu and Fan 2011). The maximum angle method seemed to determine the MLD accurately when applied to Seaglider data; however, it was suggested to be unsuitable for low-resolution profiles because of its dependence on linear regression (Chu and Fan 2011). As the shapes of the mixed layer and the underlying water are often complex as a result of the variety of dynamic processes, how to reliably determine the MLD becomes much more important.

In this study we proposed a new objective method, referred to as the "relative variance method" (Rel Var), that is based on the automated estimation, over the depth span from the surface to each profile depth, of the standard deviation normalized by the maximum variation of the temperature or density. The depth of the minimum value of the relative variance is identified as the MLD. The effectiveness of this new method is evaluated using the World Ocean Circulation Experiment (WOCE) data against the results of other available methods. The performance of the new method is indicated to be the optimal one and is less affected by data noise and depth resolution. The outline of this paper is as follows. In section 2 we describe the hydrographic data and the methodology in details. In section 3 we test the influence of the random noise on the new method and examine its performance in comparison with other available methods using some examples and the profiles of different depth resolutions of the WOCE section A05. In section 4 we present the global MLD variability determined by the threshold and objective methods. A summary is presented with a discussion in section 5 . 


\section{Data and method}

\section{a. Hydrographical data}

We used temperature and salinity data from the WOCE, the U.S. Climate Variability and Predictability Program (CLIVAR), and other programs, which were downloaded from the CLIVAR and Carbon Hydrographic Data Office website (https://cchdo.ucsd.edu/) on 9 July 2015. These data include 723 sections and 34705 stations between 2 April 1980 and 17 July 2014. The data quality is controlled by the following criteria:

- There should exist at least two data points between 10 and 40 dbar.

- The maximum measured depth is $\geq 50 \mathrm{dbar}$.

- The mean depth resolution is $\leq 2 \mathrm{dbar} ; 673$ profiles cannot meet this requirement and are ruled out. The remaining profiles have depth resolutions of 1 or 2 dbar, in particular $78.3 \%$ of them have a depth resolution of $2 \mathrm{dbar}$. As discussed below, the relative variance method is less affected by the depth resolution; however, this criterion ensures that the depth resolutions of all profiles are consistent.

- The shallowest measurement depth of a profile is $\leq 20 \mathrm{dbar}$.

- The maximum temperature (salinity, density) difference in the depth range between the sea surface and $20 \mathrm{dbar}$ should be less than the maximum respective difference in the depth range from $20 \mathrm{dbar}$ to the deepest depth of the examined profile

- The temperature difference of the whole profile is $\geq 1^{\circ} \mathrm{C}$.

These criteria leave a final dataset of 32686 CTD profiles by removing some profiles that are useless, erroneous, or contain atypical data for the MLD computation. The coverage of these profiles is shown in Fig. 1.

To assess the impact of depth resolution on the determination of the MLD, we applied different methods to the transatlantic hydrography section at $24.5^{\circ} \mathrm{N}$ in 2010 (WOCE section A05, ExpoCodes: 74DI20100106), where only one CTD cast was considered at each station and a total of 135 profiles were obtained. The temperature was recorded by Sea-Bird 911plus CTD with a sampling rate of $25 \mathrm{~Hz}$. Some raw data had spurious temperature values occurring slightly beneath the sea surface $(z<25 \mathrm{dbar})$. These unrealistic values were removed when their temperature is $0.02^{\circ} \mathrm{C}$ larger or smaller than the median temperature in the depth range between 25 and $40 \mathrm{dbar}$. Then, these corrected profiles are averaged and resampled to generate data of different depth resolutions from 0.04 to $25 \mathrm{dbar}$ for simulating different types of data products.

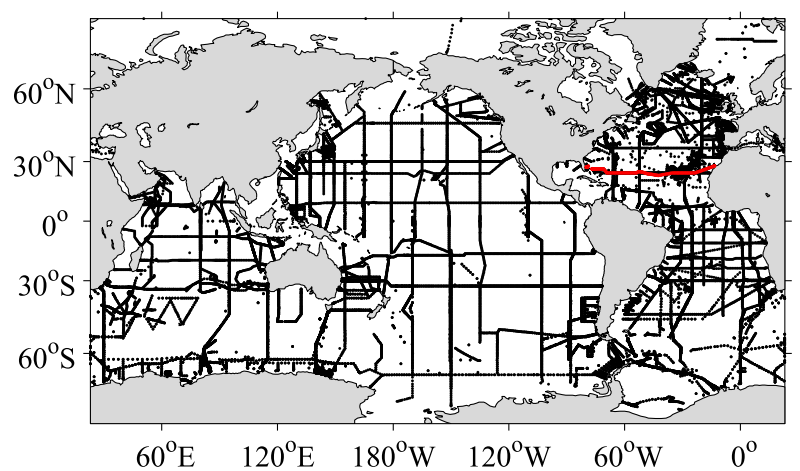

FIG. 1. Spatial distribution of WOCE data used in the study. Data are generally denser over the Atlantic Ocean. Section A05 is marked (red).

\section{b. Methodology}

As mentioned in section 1, there lies a layer of homogeneous water mixed by wind, convection, and surface flow in the ocean surface. Below the mixed layer, there is pynocline (thermocline), where the density (temperature) gradient is large. One of our motivations is to seek a universal way rather than depending upon subjective thresholds to identify the MLD. The proposed method is designed by using the fact that the range of temperature (salinity or density) is more sensitive than its variation upon the depth change around the low bound of the mixed layer. The detailed algorithm procedure for finding MLD is presented here. Let us consider a profile, $\phi(z)$, where $z$ is depth (pressure) and $\phi$ represents temperature, salinity, density, or other variables. An example, shown in Fig. 2a, is the temperature profile at station $65\left(24.5^{\circ} \mathrm{N}, 56.7^{\circ} \mathrm{W}\right)$ in section A05. For a set of points $S_{k}=\left\{z_{i}, \phi_{i}\right\}, i=1,2, \ldots, k$, ranging from the first point near the sea surface at depth $z_{1}$ to the current depth $z_{k}$, its standard deviation $\delta_{k}$ is estimated as follows:

$$
\delta_{k}=\sqrt{\frac{1}{k} \sum_{i=1}^{k}\left(\phi_{i}-\left\langle\phi_{k}\right\rangle\right)^{2}},
$$

where $\left\langle\phi_{k}\right\rangle$ is the mean value of $\phi_{i}$ over the depth range of interest $\left[z_{1}, z_{k}\right]$. While the maximum variation $\sigma_{k}$ over the same depth range is calculated by

$$
\sigma_{k}=\max _{i=1}^{k}\left(\phi_{i}\right)-\min _{i=1}^{k}\left(\phi_{i}\right)
$$

where $\max _{i=1}^{k}\left(\phi_{i}\right)$ and $\min _{i=1}^{k}\left(\phi_{i}\right)$ are the maximum and minimum values, respectively, within data points $S_{k}$. Figures $2 \mathrm{~b}, \mathrm{c}$ show the example $\delta$ and $\sigma$ profiles. Then, the relative variance of $\phi$ over the depth range $\left[z_{1}, z_{k}\right]$ is defined as

$$
\chi_{k}=\frac{\delta_{k}}{\sigma_{k}} .
$$




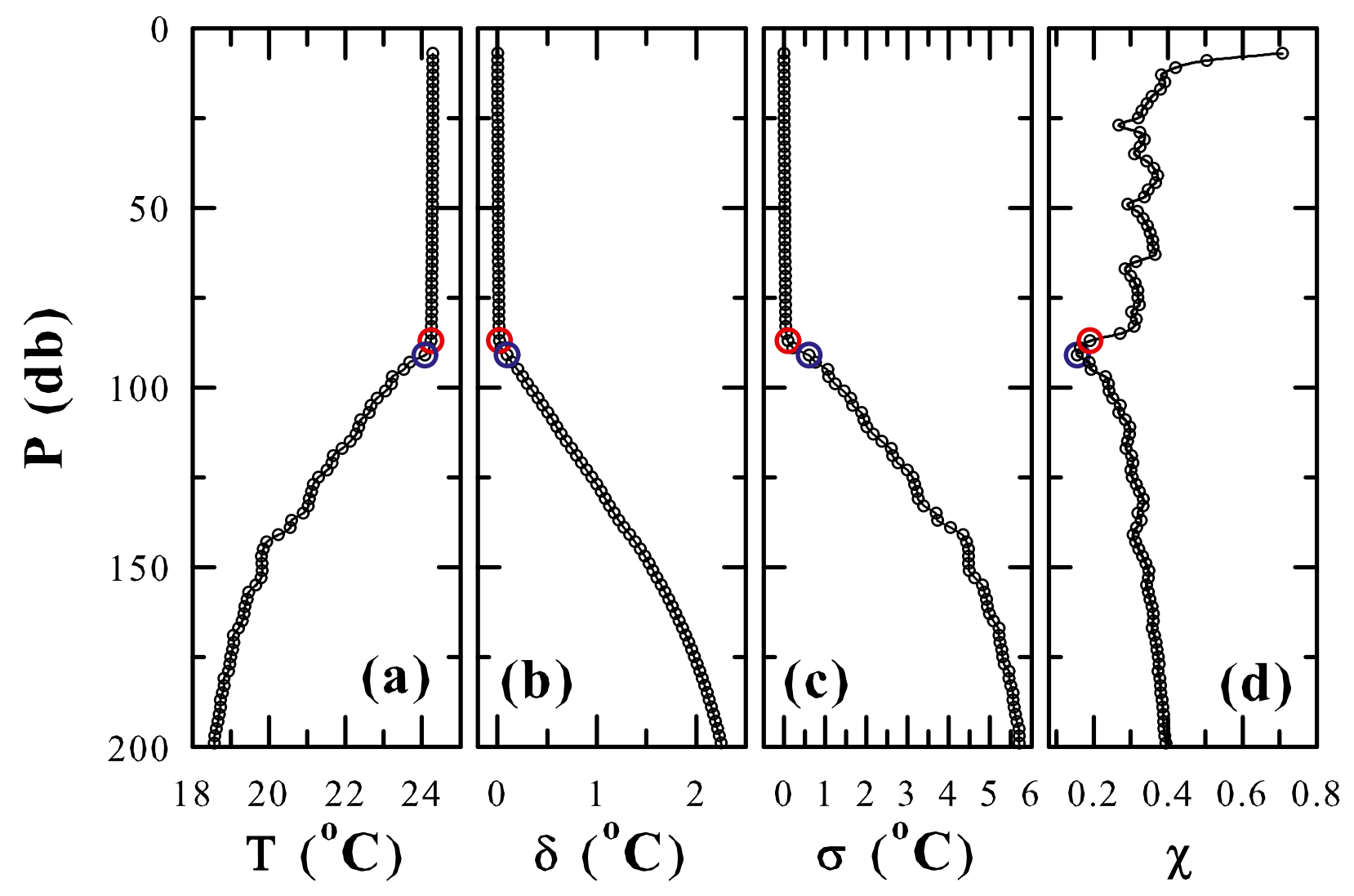

FIG. 2. Determination of MLD using the relative variance method on (a) the potential temperature profile at station $65\left(24.5^{\circ} \mathrm{N}, 56.7^{\circ} \mathrm{W}\right)$ on 23 Jan 2010 along section A05. The depth of minimum $\chi_{k}, z_{n 1}$ (blue circle) and the MLD $z_{n 2}$ (red circle) are indicated. (b)-(d) Corresponding profiles of $\delta_{k}, \sigma_{k}$, and $\chi_{k}$, respectively.

When $k$ is counted from 1 to $N$ (the total data points of the profile), the $\chi$ profile is obtained (Fig. $2 \mathrm{~d}$ ). In the case when $z_{k}$ is inside the ML, as shown in Fig. 2, both $\delta_{k}$ and $\sigma_{k}$ are less varied, which leads to a less changeable $\chi_{k}$ except for the perturbation of the first few points. While $z_{k}$ is located below the base of the ML, the variable $\phi$ changes abruptly, which results in the increased $\delta_{k}$ and $\sigma_{k}$. However, the increase of $\sigma_{k}$ is more pronounced than that of $\delta_{k}$, implying that $\sigma_{k}$ is more sensitive to the variation of $\phi$. With increasing $z_{k}, \chi_{k}$ gets ever smaller until it reaches its minimum value, the occurrence depth of which is defined as $z_{n 1}$. This procedure is described as

$$
\chi_{k} \rightarrow \min , z_{k} \rightarrow z_{n 1} .
$$

In some sense, the occurrence of the minimum $\chi_{k}$ is similar to the "misalignment problem" in the CTD measurement (Neshyba et al. 1971), where some false inversions occur at the turn points of the salinity and density profiles as a result of the different response times of the temperature and conductivity sensors. As shown in Figs. 2a-d, $z_{n 1}$ is slightly deeper than the base of the ML. Next, we trace back from $z_{n 1}$ upward to find the
MLD accurately. Here, $\delta_{k}$ is used because it is less affected by the fluctuation of $\phi$, which is indicated in the comparison between the $\delta$ and $\sigma$ profiles in Figs. 2b,c. As $\delta_{k}$ has a sharp change at the base of the ML (Fig. 2b), we can locate the corresponding $z_{n 2}$ by finding the first depth above $z_{n 1}$ for satisfying

$$
\frac{\delta_{n 2+1}-\delta_{n 2}}{\delta_{n 1+1}-\delta_{n 1}} \leq \Delta,
$$

where $\Delta$ is a critical threshold value. A $\Delta$ value between 0.1 and 0.5 works well for most of the profiles. For the profile as shown in Fig. 2, $z_{n 2}$ is changed by only 2 dbar as $\Delta$ increases from 0.1 to 0.5 . As $z_{n 2}$ is not sensitive to the selection of $\Delta, \Delta=0.3$ is selected in the present work. Then, $z_{n 2}$ is defined as the MLD, $H$.

As the ML has a limited thickness, it is much more realistic to seek the ML base at the depth $z_{m}$ of an intermediate data point rather than at the data point of the deepest depth. In practice, the variable $\phi(z)$ is detrended and the maximum or minimum value of the detrended profile is acquired depending on whether $\phi_{1}$ is larger than $\phi_{N}$. Generally, the maximum (minimum) 
value is sought for most temperature (density) profiles. In most cases, the depth of this maximum (minimum) value is close to the base of the ML, and $z_{m}$ is selected as twice of this depth. When it is impossible to define $z_{m}$ with this process, for example, to some irregular profiles, it is set as $600 \mathrm{dbar}$. This additional process reduces the chance to mislocate the MLD. Note that the detrending process is merely applied to define $z_{m}$; the proposed method is working on the original profile.

\section{The performance of the new method}

\section{a. Influence of noise}

Typically, it is unavoidable that the temperature or density profiles contain spurious spikes or inversions formed by various types of instrument noise. We will test whether the new method is sensitive to the random noise in the determination of the MLD. Here, we first construct an artificial temperature profile ranging from 0 to $300 \mathrm{dbar}$ as

$$
\tilde{T}= \begin{cases}11.8 & : 0 \leq P \leq 72 \\ 15 e^{(-P / 300)} & : 72<P \leq 300\end{cases}
$$

As shown in Fig. 3a, this artificial temperature profile is uniform for the upper $72 \mathrm{dbar}$ and is exponentially decaying with increasing depth, corresponding to an MLD of $72 \mathrm{dbar}$. Next, the temperature profile is added with the normally distributed random noise of amplitude $\tilde{T}_{\varepsilon}=\varepsilon \sigma_{300}$, where $\varepsilon$ is the noise level ranging from $0 \%$ to $5 \%$ and $\sigma_{300}$ is the maximum temperature difference in the depth range between 0 and 300 dbar. Finally, the relative variance method is applied to identify the MLD. At each noise level, 100 trials are performed and the mean and the corresponding 95\% confidence interval are shown in Fig. 3b. The random noise does not have any effect on the new determined MLD at a low noise level $(\varepsilon<0.3 \%)$. As a noise gets stronger, the determined MLD seems to gradually deviate from the expected value of $72 \mathrm{dbar}$ and the deviation reaches about $5 \mathrm{dbar}$ at $\varepsilon=5 \%$. As shown in the inset of Fig. 3a, it is clearly indicated that the temperature is indeed homogeneous in the upper $77 \mathrm{dbar}$ at $\varepsilon=5 \%$; that is, the base of MLD is moved downward as a result of the influence of random noise.

In addition, we add the normally distributed random noise to the temperature profile shown in Fig. 2a. Similar to the abovementioned artificial profile, as shown in Fig. 3d, the MLD determined by the proposed method keeps constant ( $89 \mathrm{dbar})$ at a low noise level $(\varepsilon<0.3 \%)$. The MLD slightly deviates by less than 2 dbar for the stronger noise level $(\varepsilon \leq 5 \%)$. At $\varepsilon=5 \%$, the noise amplitude reaches $0.32^{\circ} \mathrm{C}$, and it is larger than the threshold value $\left(0.2^{\circ} \mathrm{C}\right)$ used in a previous MLD work (de Boyer Montégut et al. 2004). In the influences of random noise, comparatively, it seems that the relative variance method exhibits a better performance to the real temperature profile shown in shown Fig. $3 c$ than to the artificial profile shown in Fig. 3a, which should be attributed to the fact that the water mass underneath the ML has a sharper temperature gradient in the real temperature profile. The results shown in Figs. 3b,d suggest that the relative variance method is less affected by noise and it is robust in identifying the MLD.

\section{b. Comparison with other methods}

To further evaluate the performance of the relative variance method, a comparison of this method with the six other currently used methods is being done. These methods are described as follows.

(i) Difference method (Diff): Since this method is simple and stable in determining MLD, it has been extensively applied. As recommended by de Boyer Montégut et al. (2004), the temperature difference of $0.2^{\circ} \mathrm{C}$ and the density difference of $0.03 \mathrm{~kg} \mathrm{~m}^{-3}$ (to the reference depth of $10 \mathrm{dbar}$ ) are the optimal thresholds when taking the global MLD distribution into consideration. Here, the reference depth is alternatively selected as the sea surface for consistency with the other methods.

(ii) Difference-interpolation method (Dif Int): A linear interpolation between observed levels is additionally employed to the result of the difference method to determine the MLD more accurately (de Boyer Montégut et al. 2004).

(iii) Gradient method (Grad): Following the suggestion by Dong et al. (2008), a temperature gradient criterion of $0.025^{\circ} \mathrm{Cdbar}^{-1}$ and a potential density gradient criterion of $0.0005 \mathrm{~kg} \mathrm{~m}^{-3} \mathrm{dbar}$ has been used to determine the MLD.

(iv) Hybrid method $(\mathrm{Hybr})$ : From a suite of possible MLD values assembled by the difference and gradient methods, a complicated fitting algorithm is employed to approximate the MLD (Holte and Talley 2009). The detailed algorithm of the method are publicly available.

(v) Curvature method (Curv): In this method one examines the second derivative of temperature (density) with respect to depth, referred to in the literature as "curvature" (Lorbacher et al. 2006). For each data point, a curvature value is being estimated and the first (as one scans the profile downward from the sea surface) local maximum of curvature (within some tolerance of one standard deviation of temperature/density) is being defined as the lower MLD base. The algorithm of the method is publicly available. 


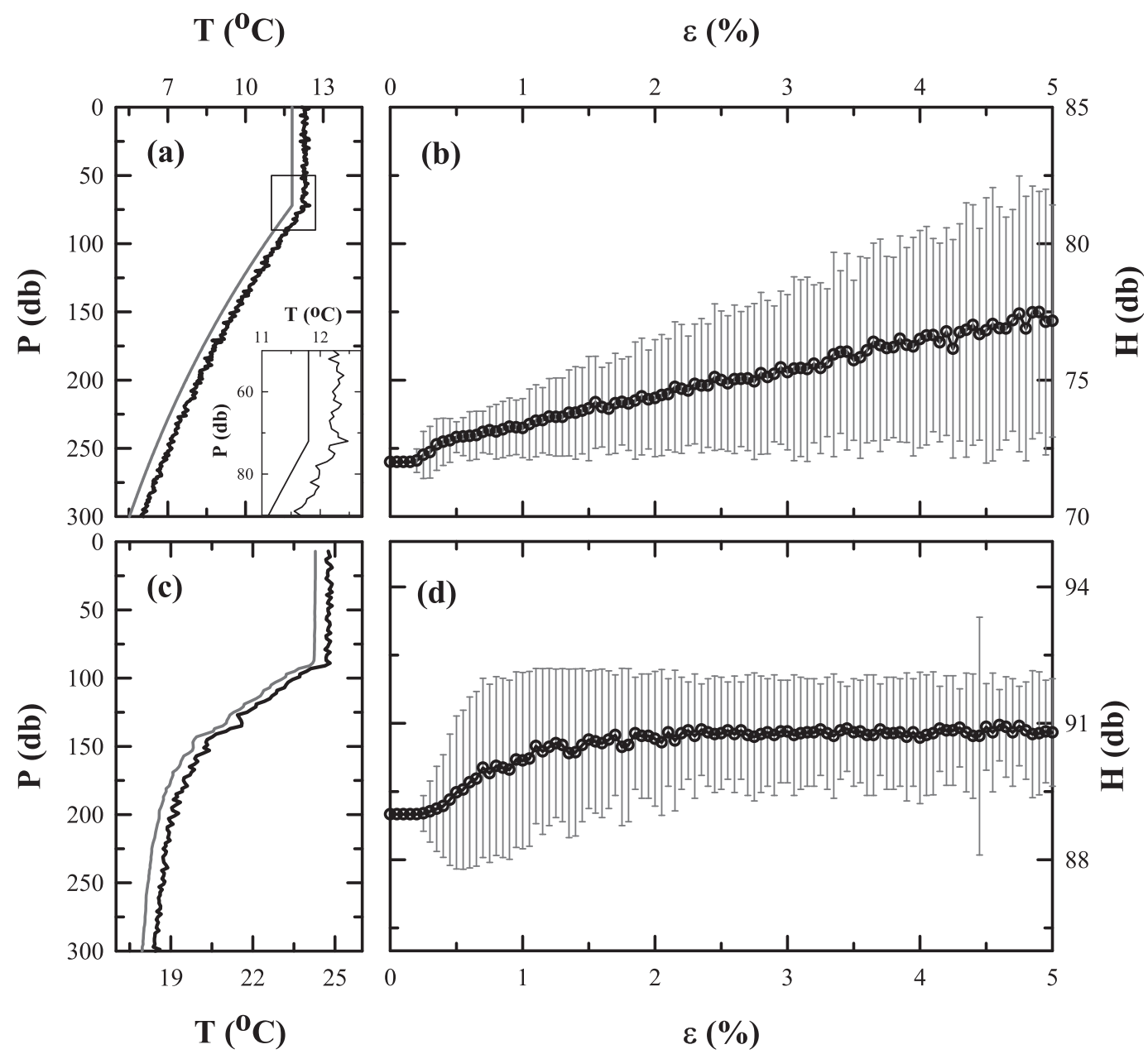

FIG. 3. (a) Artificial temperature profiles $\tilde{T}$ generated by Eq. (6) (gray line) and $\tilde{T}+\tilde{T}_{5 \%}$ (black line), where $\tilde{T}_{5 \%}$ is the normally distributed random noise at $\varepsilon=5 \%$. To avoid overlap, $\tilde{T}+\tilde{T}_{5 \%}$ is shifted by $0.5^{\circ} \mathrm{C}$. (b) The MLD $H$ of the artificial profile in (a) as a function of $\epsilon$. At each $\epsilon$, the error bar of MLD is the 95\% confidence interval over 100 trails. (c) Temperature profile as in Fig. 2a (gray line) and the constructed one (black line) with the addition of the normally distributed random temperature noise at $\varepsilon=5 \%$. To avoid overlap, the constructed temperature profile is shifted by $0.5^{\circ} \mathrm{C}$. (d) The MLD $H$ of the profile in (c), but as function of $\epsilon$. At each $\epsilon$, the fluctuation of MLD is the $95 \%$ confidence interval over 100 trails.

(vi) Maximum angle method (Max Ang): According to this method, two linear regressions are applied to the temperature/density profile, one on the data points above and one beneath each data depth. The depth, at which the angle formed by the two aforementioned linear fits is maximum, is identified as the MLD (Chu and Fan 2011).

\section{1) EXAMPLE PROFILES}

Here, we first show the efficiency of these methods in determining the MLD of four temperature profiles from the section A05. As shown in Fig. 4a (station 65), when the ML and the underneath water column are well separated, all the methods can approximately determine the MLD. The MLD is visually inspected to be $89 \mathrm{dbar}$. It is estimated to be within the range between $87.9 \mathrm{dbar}$ by the curvature method and 94.0 dbar by the difference method. When the dynamics are complex in the upper ocean, leading to highly stratified water columns occurring in several depth ranges (Fig. 4b, station 69), the determined MLDs by different methods are much scattered. The base of a homogeneous layer is identified by the hybrid, curvature, and relative variance methods at 61,65 , and $59 \mathrm{dbar}$, respectively. The difference and difference-interpolation methods also approximately capture the MLD, which are 72.0 and $70.5 \mathrm{dbar}$, respectively. This is consistent with the findings in previous studies (Lorbacher et al. 2006; Holte and Talley 2009); that is, the estimations from the difference and 


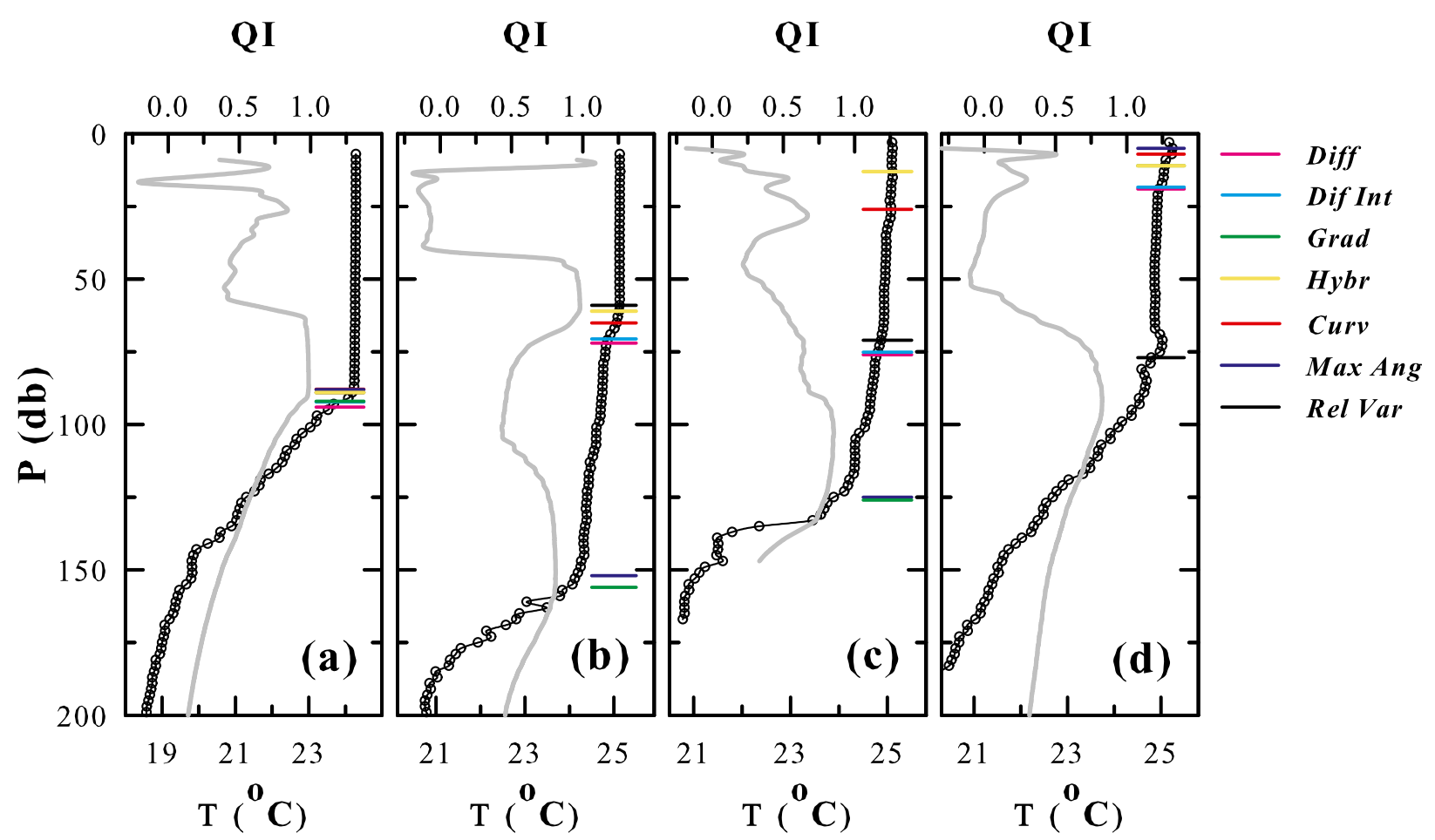

FIG. 4. Potential temperature profiles (circles with black lines) and the corresponding QI (gray lines) defined in Eq. (7b) with the vertical space $\Delta z=2 \mathrm{dbar}$ at (a) station $65\left(24.5^{\circ} \mathrm{N}, 56.7^{\circ} \mathrm{W}\right)$, (b) station $69\left(24.5^{\circ} \mathrm{N}, 53.9^{\circ} \mathrm{W}\right)$, (c) station $13\left(27.3^{\circ} \mathrm{N}, 79.2^{\circ} \mathrm{W}\right)$, and $(\mathrm{d})$ station 60 $\left(24.5^{\circ} \mathrm{N}, 60.4^{\circ} \mathrm{W}\right)$ along section A05. The MLDs $H$ evaluated using different methods are marked: the difference (pink), differenceinterpolation (cyan), gradient (green), hybrid (yellow), curvature (red), maximum angle (blue), and relative variance (black) methods.

difference-interpolation methods are generally deeper than the actual MLD. The determinations by the maximum angle and gradient methods are 152 and $156 \mathrm{dbar}$, respectively, which correspond to the top depth of deeper stratified water column. In the case where the temperature changes gradually with increasing depthfor example, in station 13 (Fig. 4c) - the hybrid method identifies the MLD as $13 \mathrm{dbar}$, which seems too shallow with visual inspection. The curvature method finds the MLD to be $26 \mathrm{dbar}$. The relative variance method finds the almost homogeneous temperature depth range with the determined MLD of 71 dbar. The MLD determined by the difference and difference-interpolation methods is decided by the threshold values, which are 76.0 and 75.2 dbar, respectively. The maximum-angle and gradient methods capture the top of the deeper stratified water column; their MLDs are estimated to be 126 and 124 dbar, respectively. When the temperature signal gets more complicated in the ML-for example, the temperature inversions occurred at station 60 (Fig. 4d) - the curvature and maximum angle methods are affected by the temperature inversion around $p=6 \mathrm{dbar}$ and they determine the MLDs to be 7 and 5 dbar, respectively. The hybrid and gradient methods find the same MLD, 11 dbar. The MLDs by the difference and difference- interpolation methods are still affected by the threshold value, being 19 and $18.4 \mathrm{dbar}$, respectively. The relative variance method looks insensitive to the temperature inversion and determines the MLD to be $77 \mathrm{dbar}$. These examples show that the relative variance and curvature methods tend to find similar MLDs for the most homogeneous layer, but that the relative variance method is less affected by the small-scale processes, for example, inversion. The difference and difference-interpolation methods give a slightly thicker MLD, and the maximum angle and gradient methods easily overestimate the MLD in most cases. The hybrid method looks to find the more reliable MLD than the difference and gradient methods, probably attributing to its assembled algorithm (Holte and Talley 2009).

To evaluate whether a method is able to cleanly separate the homogeneous layer from the stratified region below, Lorbacher et al. (2006) proposed a quality index (QI),

$$
\mathrm{QI}=1-\frac{\delta_{n 2}}{\delta_{1.5 \times n 2}}
$$

where $\delta_{n 2}$ is the standard deviation within the ML and $\delta_{1.5 \times n 2}$ is the standard deviation of $\phi$ over the depth range of 1.5 times the MLD from the sea surface. Based 
on the definition of QI, it is expected that QI approaches 1 when the ML is well developed. As reported by Lorbacher et al. (2006), QI is above 0.7 in $70 \%$ of the World Ocean. To examine whether QI can reflect the accuracy of the determined MLD, QI in Eq. (7a) is promoted to any depth $z_{k}$,

$$
\mathrm{QI}_{k}=1-\frac{\delta_{k}}{\delta_{1.5 \times k}} .
$$

In the example profile shown in Fig. 4a, it is found that QI corresponds to the maximum value when approaching the base of a highly homogeneous ML. The maximum QI $(\mathrm{QI}=0.99)$ is obtained when the MLD is determined with the highest accuracy of 89 dbar. For the profile shown in Fig. 4b, QI is larger than 0.95 for the hybrid, curvature, and relative variance methods; 0.71 for the difference method; 0.80 for the difference-interpolation method; and around 0.81 for the maximum angle and gradient methods. QI is relatively small for the profile shown in Fig. 4c: it varies from 0.52 for the hybrid method to 0.80 for the gradient method. For the profile shown in Fig. 4d, QI is 0.77 for the relative variance method, and it is less than 0.60 for the other methods. These examples show that a larger QI value generally corresponds to an MLD that is determined with higher accuracy. Recently, QI has been further refined to categorize the MLD of different quality levels with the aid of an additional parameter (Abdulla et al. 2016).

\section{2) INFLUENCE OF DEPTH RESOLUTION}

The raw temperature data in section A05, as shown in Fig. 1, were averaged and resampled to obtain profiles of different depth resolutions $\Delta z_{l} \in\{0.040 .10 .20 .5123 \ldots \ldots 25\}$ dbar (the total number of resolutions is $N_{\text {res }}=29$ ). At each station we find the MLDs of the temperature profile at different depth resolutions using different methods. As the performance of each method cannot be readily evaluated based on few individual profiles, we perform the statistical analysis on all profiles $\left(N_{\text {prof }}=135\right)$. For each method we examine the mean and standard deviation of $H$ and QI of each resolution. In particular, if $X$ refers to $H$ or QI, its mean value at the resolution $\Delta z_{l}$ is defined as

$$
<X>_{l}=\frac{1}{N_{\text {prof }}} \sum_{j=1}^{N_{\text {prof }}} X_{j, l}
$$

where $X_{j, l}$ is $X$ at station $j$ with the resolution $\Delta z_{l}$. The standard deviation of $X$ at the resolution $\Delta z_{l}$ is defined as

$$
\delta_{X_{l}}=\sqrt{\frac{1}{N_{\text {prof }}} \sum_{j=1}^{N_{\text {prof }}}\left(X_{j, l}-\langle X\rangle_{j}\right)^{2}},
$$

where $\langle X\rangle_{j}$ is the mean value of each profile over all depth resolutions, and it is expressed as

$$
<X>_{j}=\frac{1}{N_{\text {res }}} \sum_{l=1}^{N_{\text {res }}} X_{j, l} .
$$

Figure 5 shows $\langle H\rangle_{l}$-that is, the averaged MLDs (over all 135 profiles) versus the depth resolution $\Delta z_{l}$-derived with different methods (corresponding to different colors). Overlaid for comparison is also the average visually inspected MLD value of $92.4 \mathrm{dbar}$. During the visual inspection process, the temperature fluctuation $\delta$, the temperature difference $\sigma$, and QI are also considered to ensure a more reliable MLD for each profile.

In particular, the function $\langle H\rangle_{\text {l }}$ that is determined by the difference method deviates gradually from the visually inspected value and gets deepened with increasing $\Delta z_{l}$, in accord with the finding of Helber et al. (2012). The large deviation at low resolution (large $\Delta z_{l}$ value) is attributed to the fixed threshold, and this shortcoming is partially remedied by the differenceinterpolation method. Namely, as shown in Fig. 5a, although the average MLD values $\langle H\rangle_{l}$ increase with increasing $\Delta z_{l}$ for both methods (difference/difference interpolation), $\langle H\rangle_{l}$ assessed by the differenceinterpolation method presents a deviation from the reference value (i.e., the visually inspected one) that is overall smaller than the respective deviation that is derived by the difference method. On the other hand, the gradient method is most seriously affected by $\Delta z_{l}$ even though its $\langle H\rangle_{l}$ (over all $\Delta z_{l}$ values) is close to the visually inspected MLD. Comparably, the MLD determined by the difference method varies less with the depth resolutions (Fig. 5b), which is consistent with the result of Brainerd and Gregg (1995); $\left\langle H>_{l}\right.$ of the hybrid method is slightly smaller than the visually inspected one, but it remains almost unchangeable for different depth resolutions. Furthermore, $\langle H\rangle_{l}$ derived with the curvature method is much smaller and therefore deviates much more from the visually inspected value at small $\Delta z_{l}$ values, as a result of the temperature profiles containing more spikes, which greatly affect this method (Chu and Fan 2011). For the maximum angle method, $\langle H\rangle_{l}$ gradually decreases with increasing $\Delta z_{l}$, while it tends to approach the visually inspected value, at large $\Delta z_{l}$ (low depth resolution) values. This is contrary to the expected in Chu and Fan (2011), where the method was suggested to be 

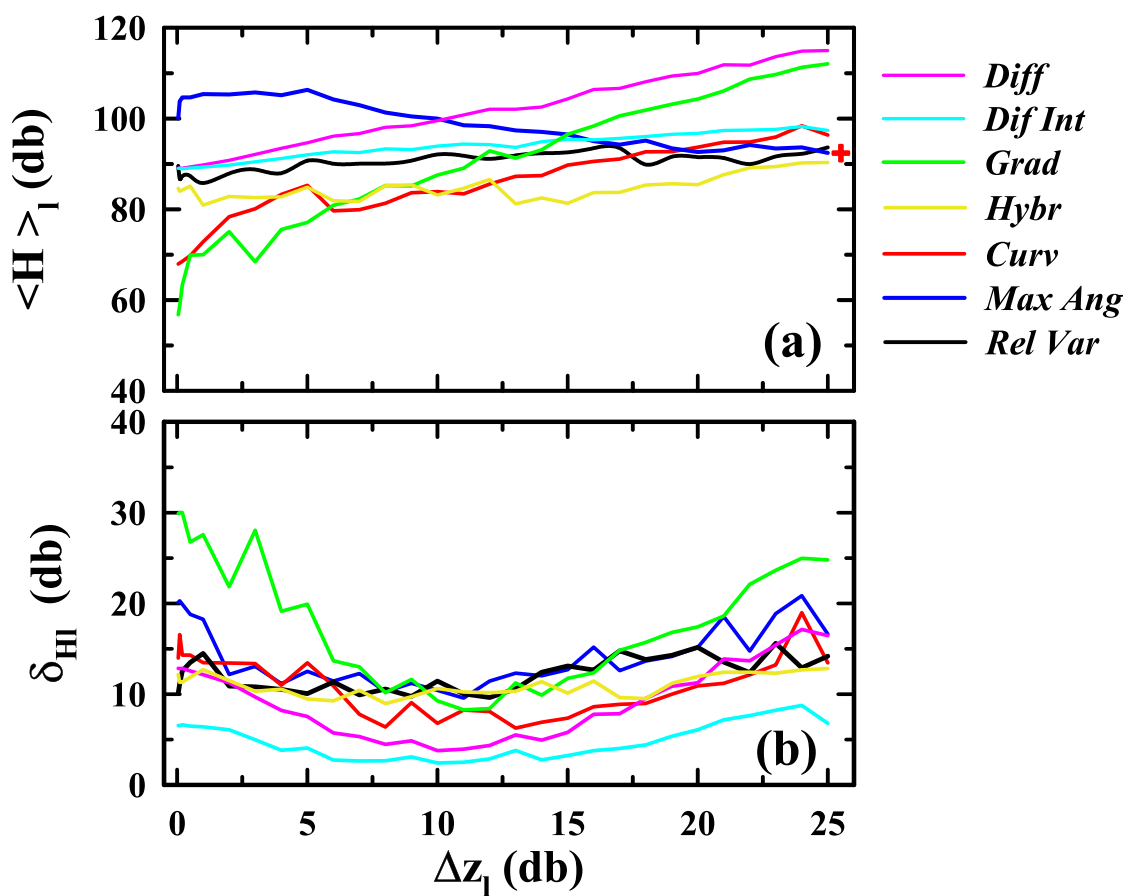

FIG. 5. (a) The mean MLD $\langle H\rangle_{l}$ of all 135 stations as a function of the vertical space $\Delta z_{l}$ by the difference (pink), difference-interpolation (cyan), gradient (green), hybrid (yellow), curvature (red), maximum angle (blue), and relative variance (black) methods. Mean MLD by visual inspection is marked (red cross). (b) As in (a), but for the standard deviation of MLD $\delta_{H l}$ as a function of $\Delta z_{l}$.

unapplicable for low depth resolution profiles. Finally, the $\langle H\rangle_{\text {l }}$ that is determined by the relative variance method presents the best agreement with the visually inspected value with considerable stability from the aforementioned reference value (Fig. 5b). The mean, median, and standard deviation values of MLDs for all resolutions, as listed in Table 1 , show consistent results with those shown in Fig. 5.

Additionally, the dependences of QI, its mean $\langle\mathrm{QI}\rangle_{l}$, and standard deviation $\delta_{\mathrm{QII}}$ on the depth resolution $\Delta z_{l}$ are shown in Fig. 6. In particular one can see in this figure that the $\langle\mathrm{QI}\rangle_{l}$ values that are derived by the difference and gradient methods gradually decrease from about 0.9 to 0.64 with increasing $\Delta z_{l}$; this fact suggests that the threshold methods cannot accurately determine the MLD, especially at low depth resolution. $\langle\mathrm{QI}\rangle_{I}$ of the hybrid method seems better, varying around 0.85 , because this method integrates both the difference and gradient methods. The shortcoming caused by the low depth resolution is greatly remedied by the interpolation in the difference-interpolation method; however, the produced $\langle\mathrm{QI}\rangle_{l}$ is still less than those of the objective methods (relative variance, curvature, and maximum angle methods). These objective methods produce larger $\langle\mathrm{QI}\rangle_{l}$; moreover, the relative variance method determines the MLD of the highest $\langle\mathrm{QI}\rangle_{l}$, which is around 0.94

TABLE 1. Statistics of $H$ and QI for all stations along the section A05, showing the corresponding mean $\langle X\rangle$, median $X_{m}$, and standard deviation $\delta_{X}$ values, with different methods. Percentage $(P)$ of all profiles with different resolutions, where the determined MLD is of QI $>0.85$ with different methods, is presented.

\begin{tabular}{lccccccc}
\hline \hline \multicolumn{1}{c}{ Method } & $\langle H\rangle_{l}(\mathrm{dbar})$ & $H_{l m}(\mathrm{dbar})$ & $\delta_{H l}(\mathrm{dbar})$ & $\langle\mathrm{QI}\rangle_{l}$ & $\mathrm{QI}_{l m}$ & $\delta_{\mathrm{QIl}}$ & $P(\%)(\mathrm{QI}>0.85)$ \\
\hline Visual inspection & 92.4 & 98.3 & & & & & \\
Difference & 100.9 & 103 & 9.40 & 0.76 & 0.82 & 0.14 & 43.4 \\
Difference interpolation & 93.6 & 96.2 & 4.90 & 0.86 & 0.95 & 0.10 & 74.2 \\
Gradient & 88.1 & 91.0 & 18.2 & 0.75 & 0.83 & 0.20 & 43.8 \\
Hybrid & 84.7 & 84.6 & 11.0 & 0.85 & 0.85 & 0.17 & 77.3 \\
Curvature & 84.7 & 90.6 & 14.5 & 0.89 & 0.97 & 0.17 & 82.9 \\
Maximum angle & 99.1 & 102.0 & 10.9 & 0.88 & 0.95 & 0.10 & 72.2 \\
Relative variance & 90.5 & 93.0 & 12.3 & 0.94 & 0.97 & 0.05 & 88.8 \\
\hline
\end{tabular}




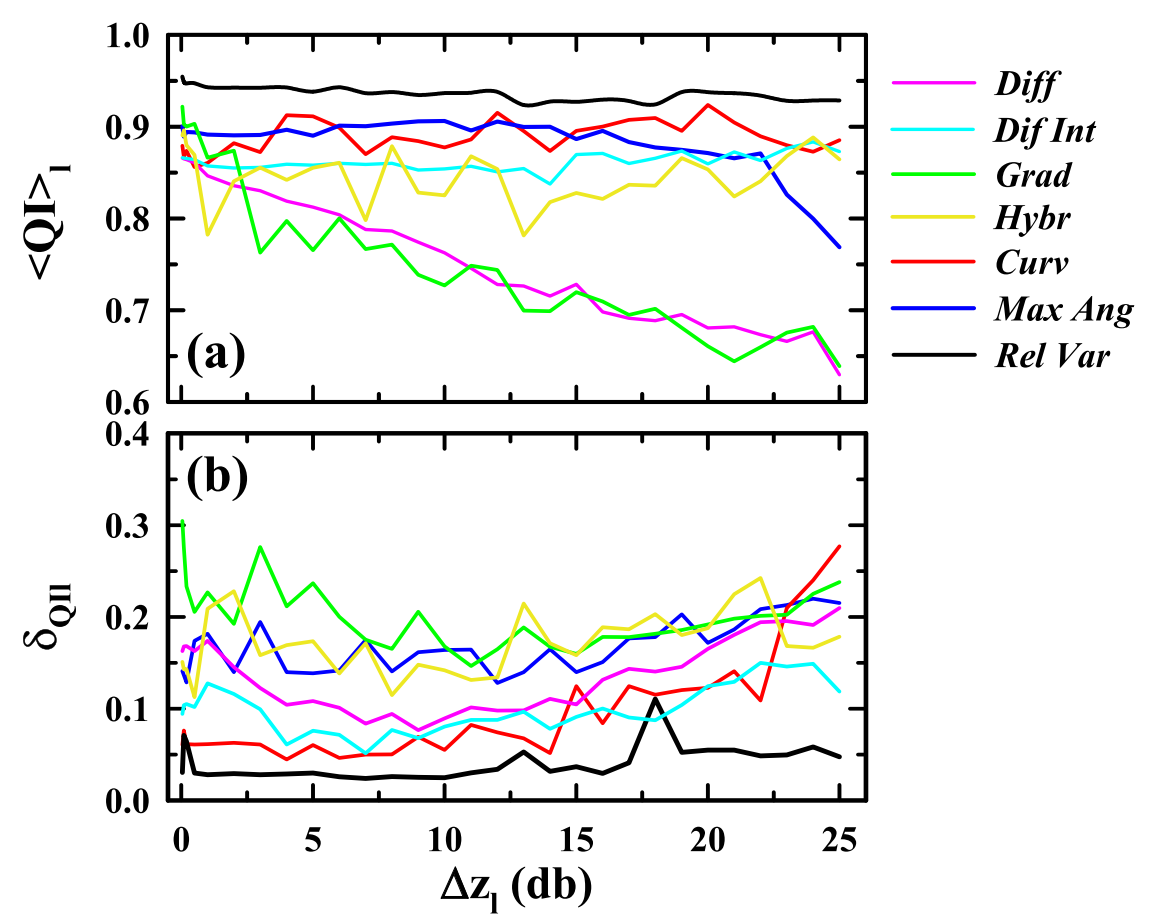

FIG. 6. (a) The mean quality index $\langle\mathrm{QI}\rangle_{l}$ of all 135 stations as a function of the vertical space $\Delta z_{l}$ by the difference (pink), difference-interpolation (cyan), gradient (green), hybrid (yellow), curvature (red), maximum angle (blue), and relative variance (black) methods. (b) As in (a), but for the standard deviation of quality index $\delta_{\mathrm{QIl}}$ as a function of $\Delta z_{l}$.

(Table 1). As indicated in Fig. $6 \mathrm{~b}$ about $\delta_{\mathrm{QII}}$, the differenceinterpolation method is less varied compared to the difference and gradient methods, its stability is close to that of the curvature method, and it is better than that of the maximum angle method. Comparatively, the relative variance method has the smallest $\delta_{\text {QII }}(\sim 0.05)$ and the gradient method has the largest $\delta_{\text {QII }}(\sim 0.2)$. Furthermore, it can be seen in the sixth column of Table 1 that $43 \%$, $74 \%, 44 \%, 77 \%, 83 \%$, $72 \%$, and $89 \%$ of the stations along the section A05 have $\mathrm{QI}>0.85$ for the difference, difference-interpolation, gradient, hybrid, curvature, maximum angle, and relative variance methods, respectively. Obviously, the hereby introduced method of relative variance is the most reliable of all methods, as it corresponds to the largest of the aforementioned percentage values $(89 \%)$. This conclusion is consistent with that drawn from the MLD results shown in Fig.5. The difference-interpolation method also works well in determining the MLD, but its small $<$ QI $>$ value implies that this method merely gives a coarse representation of the actual MLD.

\section{Application to the global ocean}

The new method is applied to the WOCE data shown in Fig. 1 to exhibit the global MLD distribution. Both temperature and density profiles are considered to determine MLD values, referred to as $H_{T}$ and $H_{\rho}$, respectively (the respective quality indices are referred to as $\mathrm{QI}_{T}$ and $\left.\mathrm{QI}_{\rho}\right)$. Three more methods are used for comparison. The difference-interpolation method is used extensively and is included here (de Boyer Montégut et al. 2004). The hybrid method of Holte and Talley (2009) is used here because it determines the MLD by optimizing the results of the difference and gradient methods. Both of them depend on threshold values. As with the relative variance method, the curvature method (Lorbacher et al. 2006) is also relatively objective in the sense that it is much less dependent on threshold values. As listed in Table 1, the difference-interpolation and hybrid methods are threshold methods of better performance, while the curvature and relative variance methods are the better ones in the objective methods.

\section{a. Probability density functions of global MLD}

The probability density functions (PDFs) of the MLD from $H_{T}$ and $H_{\rho}$ are shown in Fig. 7. One of the remarkable features in this figure is that the PDFs of $H_{T}$ and $H_{\rho}$ derived by different methods tend to coincide when $H_{T}$ and $H_{\rho}$ are approximately larger than 20 dbar, a fact implying that the MLD determined by all the aforementioned methods follows the same 


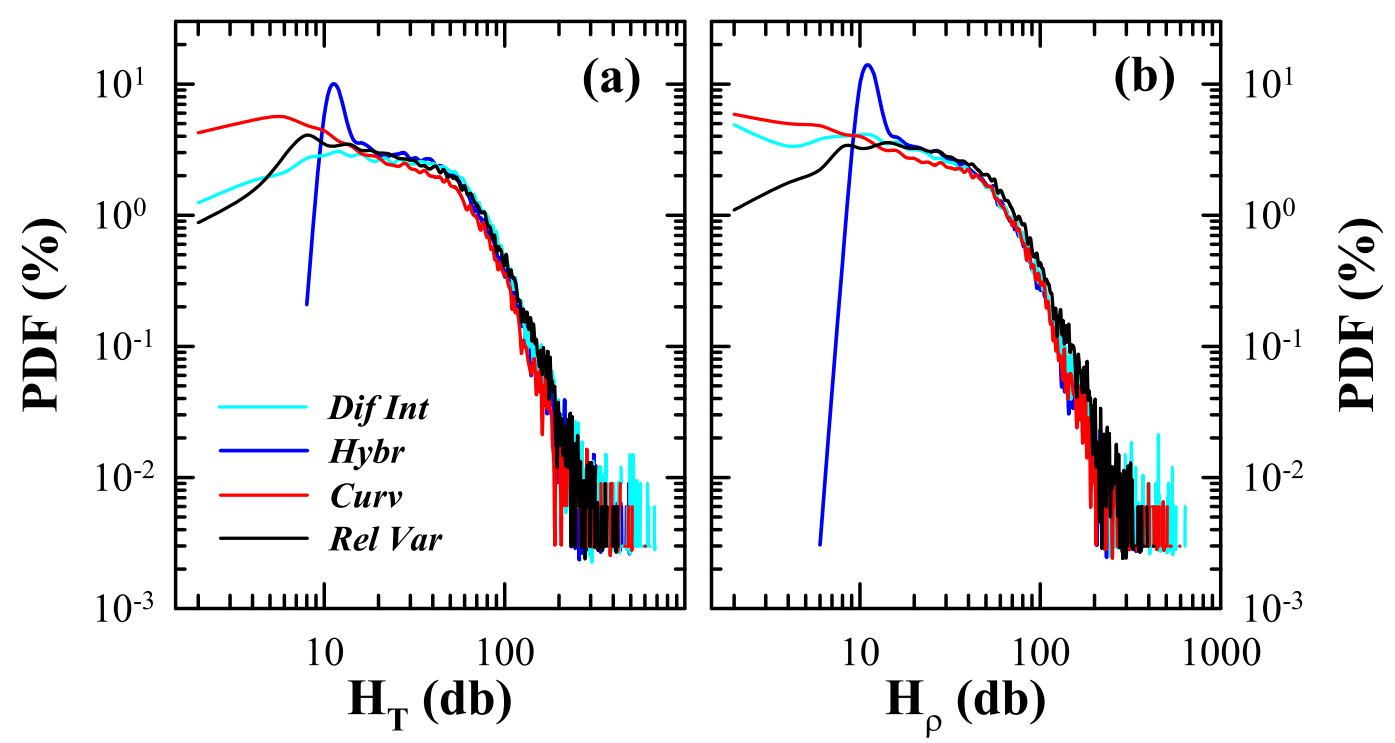

FIG. 7. PDFs of MLD (a) $H_{T}$ and (b) $H_{\rho}$ from temperature and density profiles using the difference-interpolation (cyan), hybrid (blue), curvature (red), and relative variance (black) methods.

PDF distribution globally in cases of relatively thick ML. As temperature/density close to the sea surface is easily affected by the diurnal heating cycle and by mass and momentum exchanges with the atmosphere (Price et al. 1986), some MLDs shallower than 10 dbar are less convincing because only very few data points are included within the ML, especially for these WOCE profiles of depth resolution of 2 dbar. Figure $7 \mathrm{a}$ indicates that the difference-interpolation and curvature methods are prone to finding anomalously shallow MLDs. As suggested by Lorbacher et al. (2006) and Chu and Fan (2011), the curvature method suffers from abnormal spikes, which might be responsible for the shallower MLD assessed with this method. The hybrid method produces $H_{T}$ and $H_{\rho}$ with peaks around $11 \mathrm{dbar}$ (Figs. 7a,b), possibly attributed to the artificial limits imposed in the calculating procedure (Holte and Talley 2009). The relative variance method on the other hand derives the least probable (with the smallest population) shallow MLDs in comparison with the other aforementioned methods. This can be partially explained by the fact that the method is less affected by the noise signal, for example, temperature inversions in Fig. 4d. Although the PDFs of $H_{T}$ and $H_{\rho}$ of each method have similar distributions, as shown in Fig. 7, they still are distinct when comparing the respective mean values, $<H_{T}>$ and $\left\langle H_{\rho}\right\rangle$, and median values, $H_{T m}$ and $H_{\rho m}$. As listed in Table $2,\left\langle H_{T}\right\rangle$ is 10 and 6 dbar larger than $\left\langle H_{\rho}\right\rangle$ for the difference-interpolation and hybrid methods, respectively; and $\left\langle H_{T}\right\rangle$ is 1 or 2 dbar larger than $\left\langle H_{\rho}>\right.$ for both the curvature and relative variance methods.
Figure 8 shows the PDFs of the MLD difference, $H_{T}-H_{\rho}$, estimated by different methods. The PDF of $H_{T}-H_{\rho}$ peaks at zero (within $\pm 1 \mathrm{dbar}$ ) in $20 \%$ (difference interpolation), 32\% (hybrid), 40\% (curvature), and $42 \%$ (relative variance) of the profiles. Moreover, if one loosens the criterion of coincidence of $H_{T}$ and $H_{\rho}$ by defining that $H_{T}$ and $H_{\rho}$ are identical when $\left|H_{T}-H_{\rho}\right| \leq 10$ dbar, then one derives that the $H_{T}$ and $H_{\rho}$ values that are determined by the differenceinterpolation, hybrid, curvature, and relative variance methods are identical for $69 \%, 76 \%, 81 \%$, and $79 \%$ of the profiles, respectively. These results suggest that $H_{T}$ is close to $H_{\rho}$ for most of the profiles and that this feature can be captured by all four methods. When we take a closer look at $96 \%$ of the total profiles around the PDF peak, as shown in Fig. 8b, the PDF symmetry of $H_{T}-H_{\rho}$ is different for different methods. It clearly shows that $H_{T}-H_{\rho}$ is skewed toward the positive values for the difference-interpolation and hybrid methods, with a skewness of 2.55 and 2.26, respectively. Traditionally, a barrier layer is identified by a deeper $H_{T}$ than $H_{\rho}$, while a compensated layer is defined when $H_{\rho}$ is larger than $H_{T}$ (Sprintall and Tomczak 1992; de Boyer Montégut et al. 2004). The results shown in Fig. 8b imply that the compensated layers are much less common than the barrier layers with these WOCE data, which is consistent to the global ocean results from the threshold methods (Helber et al. 2012). However, $H_{T}-H_{\rho}$ is found to be relatively symmetric for the curvature and relative variance methods, as confirmed by the relatively much smaller values of skewness $(0.39$ and 0.40 , respectively). As reported in Lorbacher et al. (2006), the 
TABLE 2. Global statistics (mean $\left\langle X>\right.$ and median values $X_{m}$ ) of $H$ and QI determined by using difference-interpolation, hybrid, curvature, and relative variance methods on the WOCE data. Percentages $-P_{T}$ and $P_{\rho}$ for the total temperature and density WOCE data, respectively - are presented in the cases that the determined MLD of QI $>0.8$ and QI $>0.5$ from these methods.

\begin{tabular}{lcccc}
\hline \multicolumn{1}{c}{ Method } & Difference interpolation & Hybrid & Curvature & Relative variance \\
\hline$\left\langle H_{T}\right\rangle$ dbar & 49.7 & 44.9 & 37.6 & 46.6 \\
$H_{T m}$ dbar & 40.0 & 36 & 27.9 & 37 \\
$\left.<H_{\rho}\right\rangle$ dbar & 39.8 & 38.7 & 35.8 & 44.9 \\
$H_{\rho m}$ dbar & 29.0 & 29 & 27.0 & 36 \\
$\left\langle\mathrm{QI}_{T}\right\rangle$ & 0.77 & 0.75 & 0.83 & 0.87 \\
$\mathrm{QI}_{T m}$ & 0.87 & 0.87 & 0.94 & 0.94 \\
$<\mathrm{QI}_{\rho}>$ & 0.74 & 0.72 & 0.84 & 0.83 \\
$\mathrm{QI}_{\rho m}(\mathrm{QI}>0.8)$ & 0.86 & 60 & 0.86 & 0.92 \\
$P_{T}(\%)(\mathrm{QI}>0.8)$ & 60 & 58 & 72 & 74 \\
$P_{\rho}(\%)(\mathrm{QI}>0.5)$ & 56 & 83 & 86 & 94 \\
$P_{T}(\%)(\mathrm{QI}>0.5)$ & 82 & 78 & 83 & 92 \\
$P_{\rho}(\%)(7$ & & & \\
\hline
\end{tabular}

curvature method tends to capture the depth of the top of a barrier layer, while the difference-interpolation method finds the depth of the top of a thermocline. As an objective method, the relative variance method tends to detect the top of a barrier layer in a temperature profile too. An example of a barrier layer is shown in Fig. 9. It was measured in the equatorial Atlantic Ocean $\left(4.3^{\circ} \mathrm{S}, 34.8^{\circ} \mathrm{W}\right)$ on 21 October 1990 . Because of the temperature inversion, a barrier layer exists slightly below the depth of $70 \mathrm{dbar}$, corresponding to the actual MLD. The relative variance and curvature methods identify this depth from both temperature and density profiles. While the difference-interpolation and hybrid methods can capture only the MLD when using the density profile (Fig. 9b), they give much deeper MLDs when using the temperature profile (Fig. 9a).
Figure 10 shows the PDFs of $\mathrm{QI}_{T}$ and $\mathrm{QI}_{\rho}$ by four different methods. The PDF peaks around 0.98, implying that these methods are capable of determining the MLD for most of the profiles. The mean, median, and other values of QI with these methods are listed in Table 2. Generally, it indicates that the QI of the objective methods (curvature and relative variance methods) is larger than those of the threshold methods (difference-interpolation and hybrid methods). Postulating that the MLD is accurately defined when its QI $>0.80$ (Lorbacher et al. 2006; Abdulla et al. 2016), the difference-interpolation, hybrid, curvature, and relative variance methods can obtain reliable MLDs from $60 \%, 60 \%, 72 \%$, and $80 \%$ of total temperature profiles, respectively. Lorbacher et al. (2006) suggested that ML interpretation

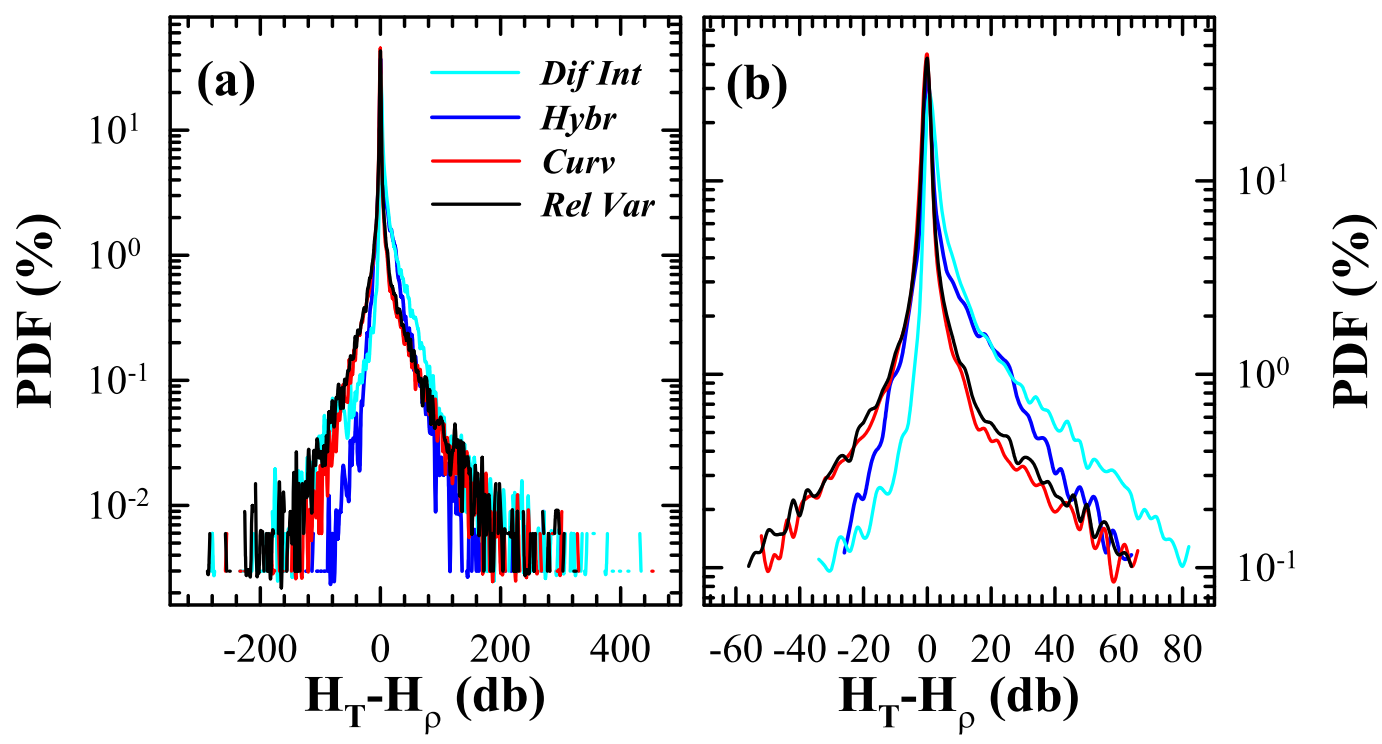

FIG. 8. (a) PDFs of MLD difference, $H_{T}-H_{\rho}$, using the difference-interpolation (cyan), hybrid (blue), curvature (red), and relative variance (black) methods. (b) As in (a), except for zooming in on the PDF peaks. 


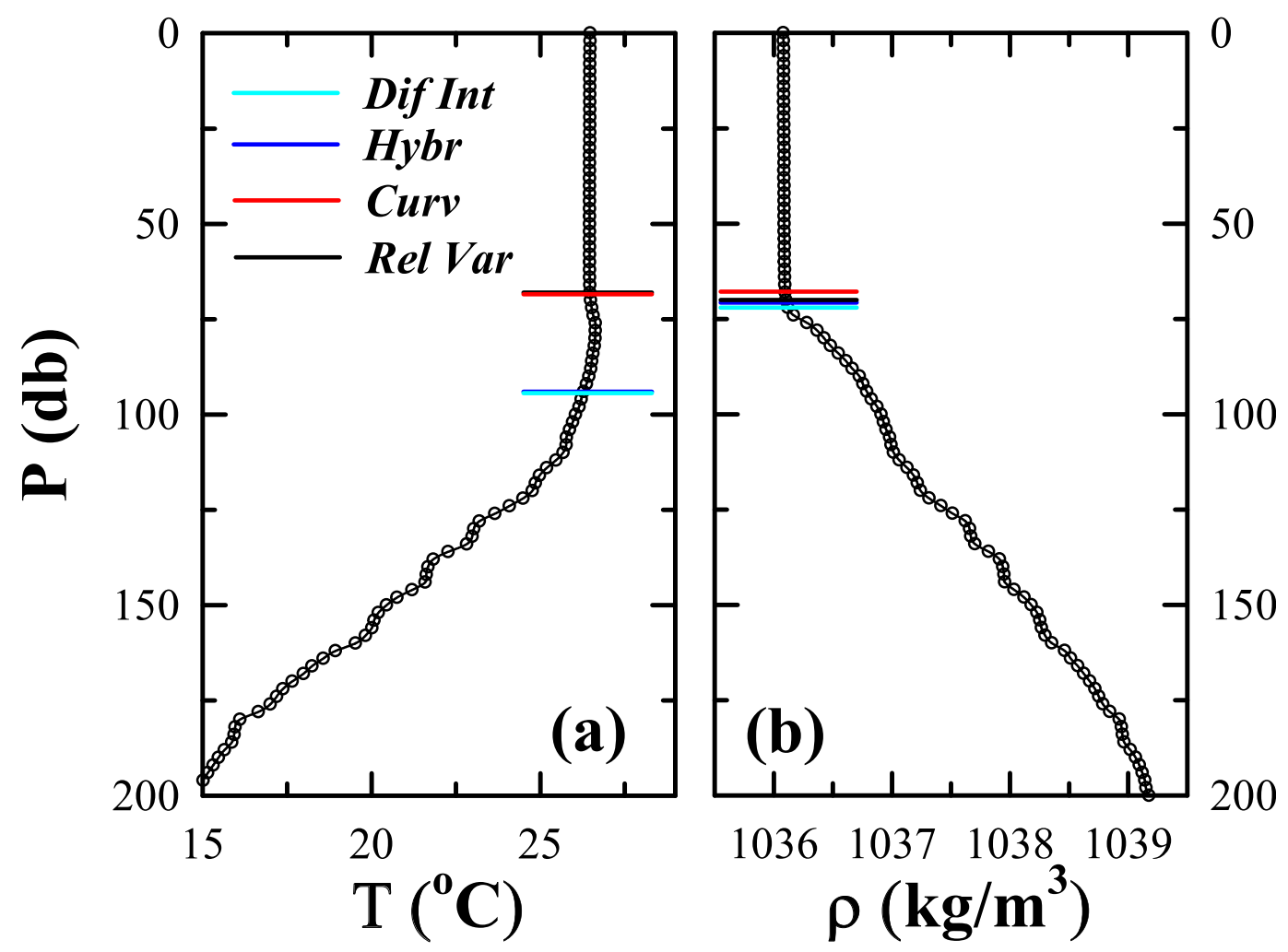

FIG. 9. Example profiles of (a) potential temperature and (b) potential density from the equatorial Atlantic Ocean $\left(4.3^{\circ} \mathrm{S}, 34.8^{\circ} \mathrm{W}\right)$ on 21 Oct 1990 . Difference-interpolation (cyan), hybrid (blue), curvature (red), and relative variance (black) methods produce MLDs of (a) 94, 94.15, 68.5, and $68 \mathrm{dbar}$, and (b) 72, 70.7, 67.8, and $70 \mathrm{dbar}$.

is impossible when $\mathrm{QI}<0.50$. The differenceinterpolation, hybrid, curvature, and relative variance methods can find the MLD of QI $>0.50$ for $82 \%, 83 \%$, $86 \%$, and $94 \%$ of the total temperature profiles, respectively. Similar results can be found from the comparison among these methods in the QI of $H_{\rho}$ (Fig. 10b; Table 2). Hence, the relative variance method gives relatively reliable results (with QI $>0.5$ ) for most

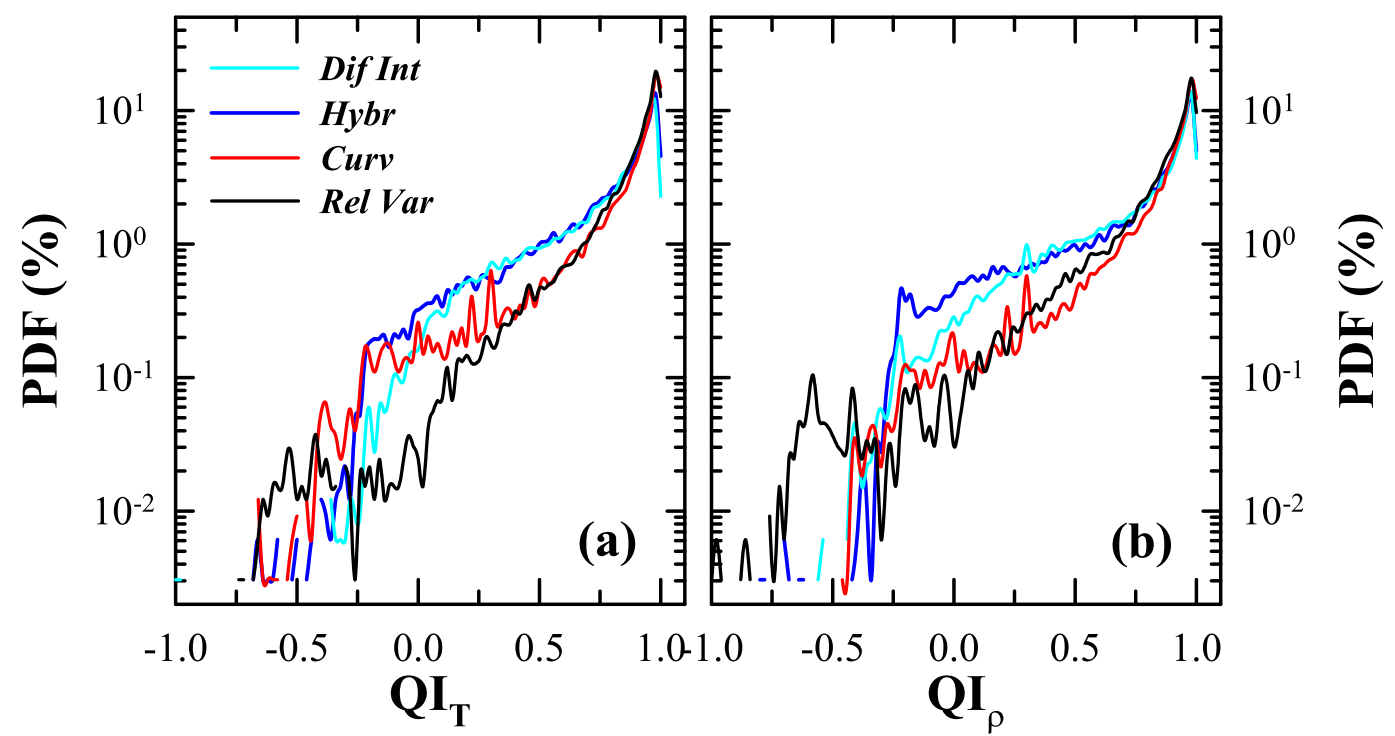

FIG. 10. PDFs of QI from (a) temperature and (b) density profiles with the difference-interpolation (cyan), hybrid (blue), curvature (red), and relative variance (black) methods. 
a) $H_{T}-$ Dif Int

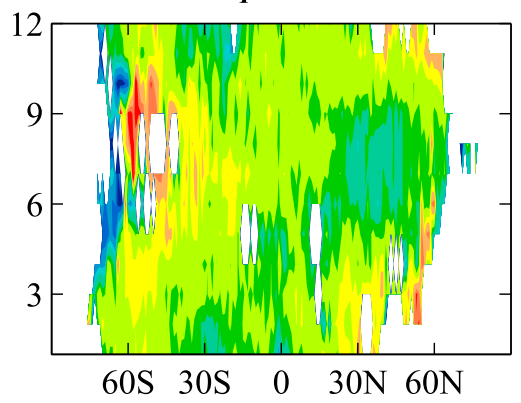

c) $\mathrm{H}_{T}-\mathrm{Hybr}$

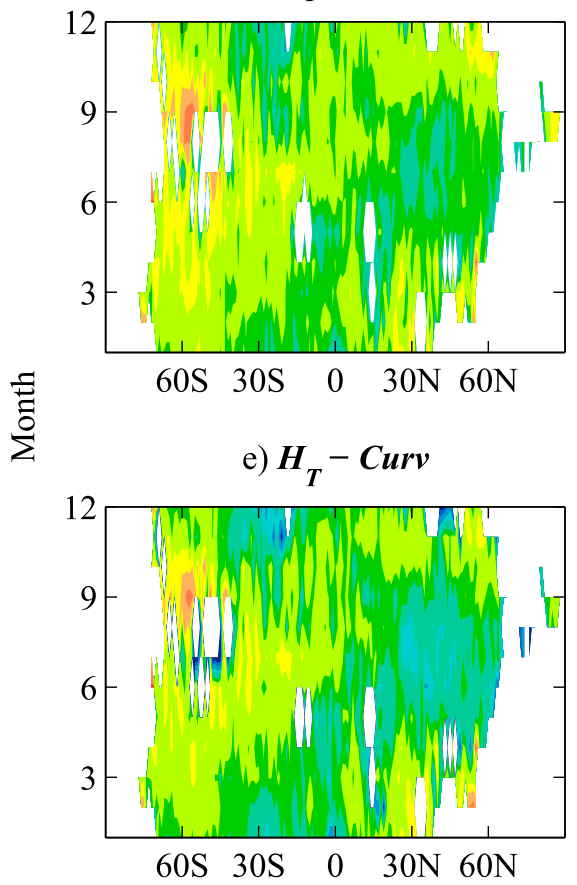

g) $\boldsymbol{H}_{T}-$ Rel Var

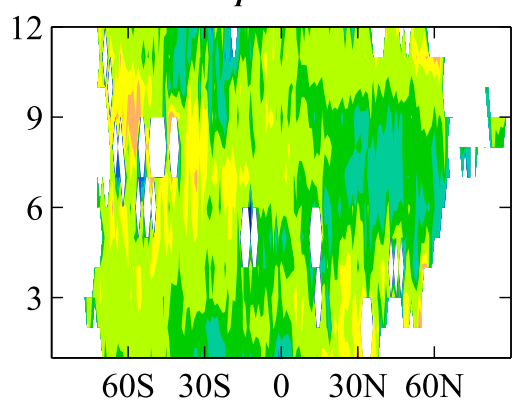

b) $H_{\rho}-$ Dif Int

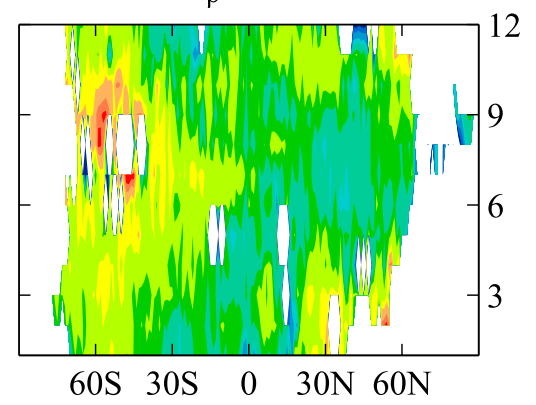

d) $\boldsymbol{H}_{\rho}-\boldsymbol{H y b r}$

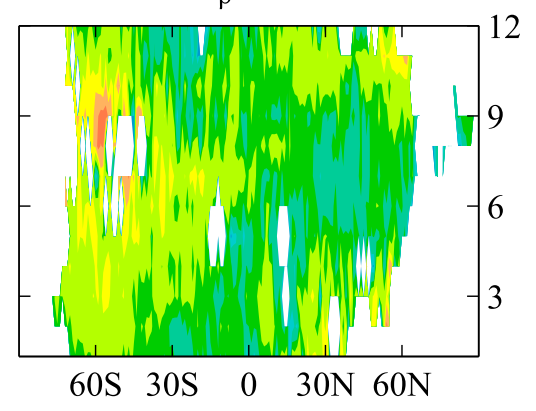

f) $\boldsymbol{H}_{\rho}-\mathrm{Curv}$

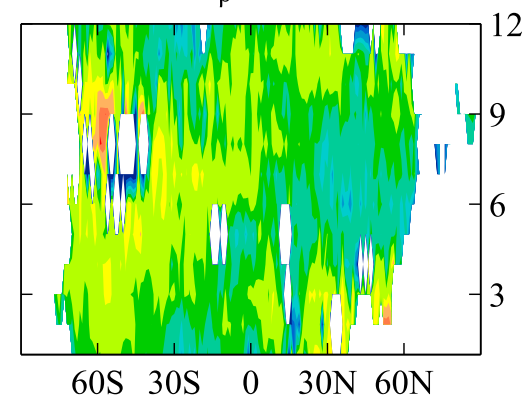

h) $\boldsymbol{H}_{\rho}-\boldsymbol{R e l}$ Var

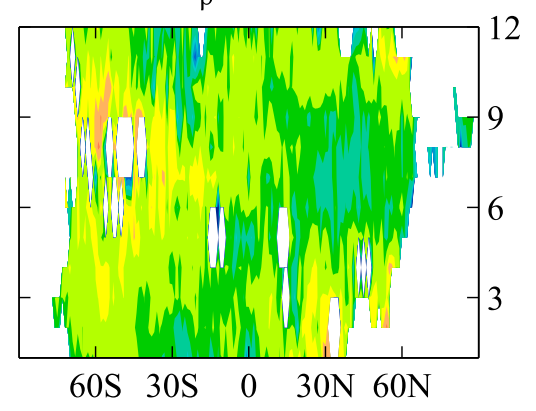

$\log _{10} H(d b)$

\section{$\begin{array}{llllll}0.5 & 1 & 1.5 & 2 & 2.5 & 3\end{array}$}

FIG. 11. Monthly variations of zonally integrated MLD, $H$, from temperature and density profiles with different methods. (a), (c), (e), (g) $H_{T}$ and (b), (d), (f), (h) $H_{\rho}$ are determined by using the difference-interpolation, hybrid, curvature, and relative variance methods, respectively. The blank regions indicate impossibility of reasonable interpolation owing to the data sparsity. 
(nearly all) profiles, thereby proving its superior effectiveness in determining the MLD.

\section{b. Global MLD variability}

To gain further insight into the effectiveness of the relative variance method (as compared to the effectiveness of the other methods) in accurately determining MLDs, it is worthwhile to additionally examine both the temporal evolution and the spatial distribution of the various MLD estimates. As indicated in Fig. 1, the WOCE data are relatively sparse in the global ocean; hence, a zonal mean is taken. Since the MLDs are characterized by strong seasonal variation, they are further averaged into monthly bins.

Figure 11 shows the spatial and temporal variation of the MLDs estimated by each of the four aforementioned methods. The blank regions indicate the impossibility of reasonable interpolation owing to data sparsity. In particular, we can see that the month-latitude patterns of MLD are similar for the four methods. In these patterns the seasonality of the MLD is well exhibited, and the seasonal variations of the MLD are almost opposite in the northern and southern hemispheres (as expected). The MLD maxima are found in the wintertime between $45^{\circ}$ and $60^{\circ} \mathrm{S}$, including the Southern Ocean, and between $30^{\circ}$ and $60^{\circ} \mathrm{N}$ corresponding to the North Atlantic Deep Water formation region. The MLD minima are found in the summertime midlatitude regions. The seasonal cycle of MLD in the region of the Antarctic Circumpolar Current is especially pronounced with several hundreds of decibars in winter and dozens of decibars in summer. Moreover, the pattern of temperature-based MLD, $H_{T}$ is similar to that of density-based MLD, $H_{\rho}$ for each method. The corresponding correlation coefficient is found to be $0.76,0.93,0.85$, and 0.89 for the difference-interpolation, hybrid, curvature, and relative variance methods, respectively. The differenceinterpolation method produces a thicker $H_{T}$ than $H_{\rho}$ (Figs. 11a,b), especially in the equatorial region, where the barrier layer prevails. This may be responsible for the low correlation of the patterns between $H_{T}$ and $H_{\rho}$ determined by this method. The difference-interpolation method finds the anomalous shallow $H_{T}$ in some regions in the wintertime MLD of the Southern Ocean, where the temperature threshold of $0.2^{\circ} \mathrm{C}$ might be inappropriate. The hybrid method produces matching patterns of $H_{T}$ and $H_{\rho}$ (Figs. 11c,d) because of its algorithm in the fitting of the ML and its underlying water column (Holte and Talley 2009). The averaged $H_{T}$ and $H_{\rho}$ from the relative variance method have very highly correlated patterns (Figs. 11g,h), which further confirm that this method is capable of accurately determining the MLD from temperature profiles even in the presence of a barrier layer. In the summertime, the MLD determined by the curvature method is shallower than those by the other methods, as is also indicated by the statistics in Table 2 .

Figure 12 shows the spatial and temporal variation of QI from different methods. Lorbacher et al. (2006) suggested that QI has large values in the hemispheric summer and fall when a sharp gradient at the base of the ML is present. This feature can be observed in the $\mathrm{QI}_{T}$ and $\mathrm{QI}_{\rho}$ of these methods. A large QI occurs from January to June in the Southern Hemisphere, and from July to November in the Northern Hemisphere. However, the difference-interpolation and hybrid methods occasionally produce low QI values even in summer and fall, especially for $\mathrm{QI}_{\rho}$ (Figs. 12b,d). This can be partly explained by the fact that the threshold methods find only the coarse MLD. Comparatively, the relative variance and curvature methods lead to larger QI values. Figure 12 also shows the QI values are generally less than 0.8 with these methods in the wintertime of the mid- and high-latitude regions, implying that the MLDs of these associated profiles are determined with considerable uncertainty. Even so, the patterns of $\mathrm{QI}_{T}$ and $\mathrm{QI}_{\rho}$ show that the objective methods (the curvature and relative variance methods) have better performance in determining the MLD.

\section{Summary and discussion}

In this study a new objective method-the relative variance method-is presented for assessing MLDs on the basis of individual temperature or density profiles. According to this method, in particular, the relative variance is the ratio where the numerator is the standard deviation value of the property (temperature, salinity) over the depth regime ranging from the surface to the "current" depth, and the denominator is the difference between the maximum and the minimum property values over the abovementioned depth regime; the MLD is defined as the depth at which the relative variance is at its minimum. For evaluating the effectiveness of the new method, the influence of random noise is examined and a comparison of this method with other available methods is conducted, including the threshold methods [difference, difference-interpolation (de Boyer Montégut et al. 2004), gradient (Dong et al. 2008), and hybrid (Holte and Talley 2009) methods] and the objective methods [curvature (Lorbacher et al. 2006) and maximum angle (Chu and Fan 2011) methods]. All methods are applied on an affluence of WOCE data.

When the example temperature profile is added with random noise (noise level $\leq 5 \%$ ), the MLD determined 
a) $Q I_{T}-$ Dif Int

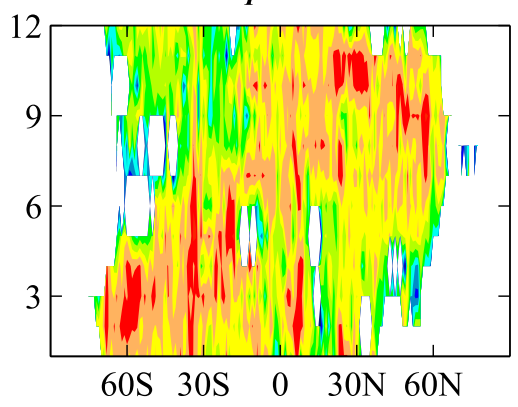

c) $Q I_{T}-\mathrm{Hybr}$
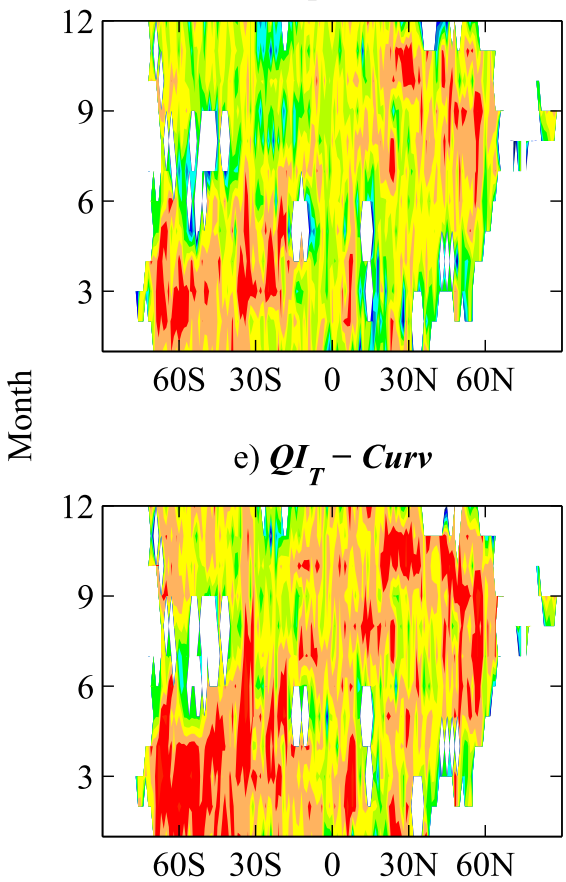

g) $Q I_{T}-$ Rel Var

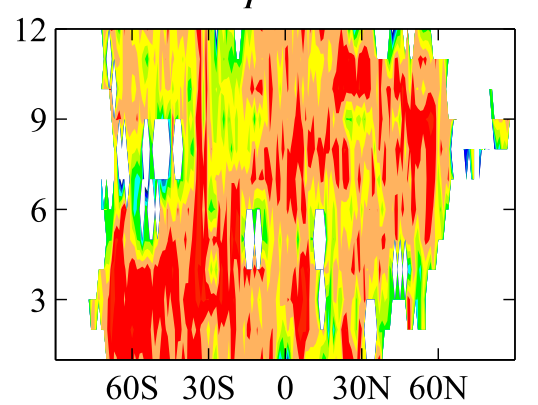

$60 \mathrm{~S} 30 \mathrm{~S} \quad 0 \quad 30 \mathrm{~N} 60 \mathrm{~N}$ b) $Q I-$ Dif Int

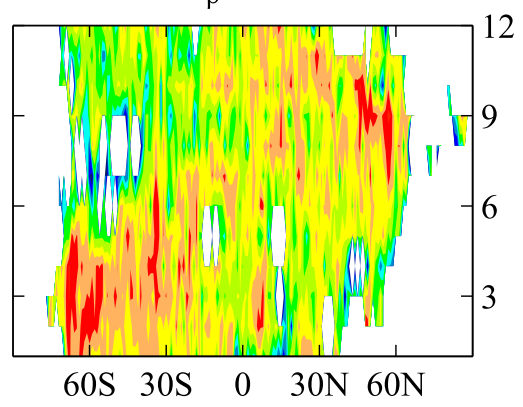

d) $Q I-H y b r$

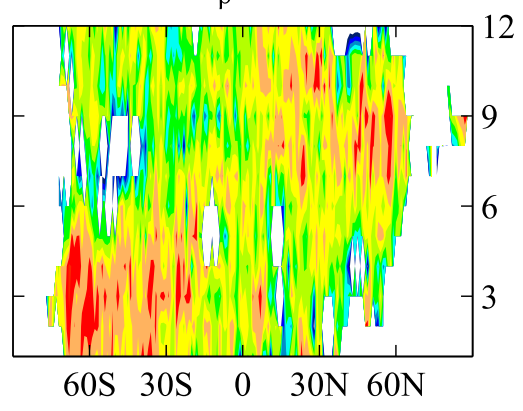

f) $Q I-C u r v$

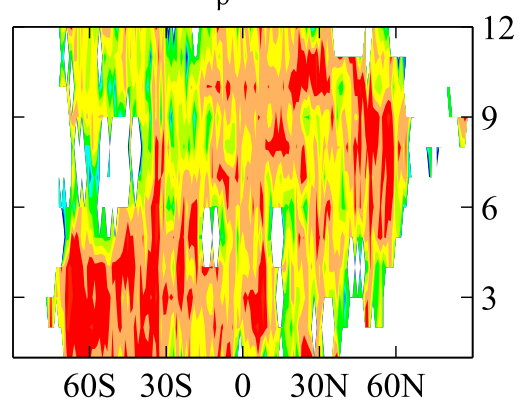

h) $\boldsymbol{Q I} I_{\rho}-\operatorname{Rel} \mathrm{Var}$

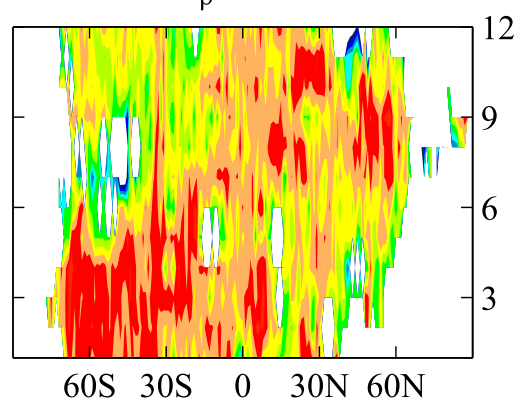

$Q I$

FIG. 12. Monthly variations of zonally integrated QI from temperature and density profiles with different methods. (a), (c), (e), (g) $\mathrm{QI}_{T}$ and (b), (d), (f), (h) $\mathrm{QI}_{\rho}$ are determined by using the difference-interpolation, hybrid, curvature, and relative variance methods, respectively. The blank regions indicate impossibility of reasonable interpolation owing to the data sparsity. 
by the new method is changed less than 2 dbar even the noise amplitude is as large as $0.32^{\circ} \mathrm{C}$ (Fig. 3d). The profiles are often contaminated by instrument noises, measurement errors, and ship heave motions, which often lead to spurious inversions or spikes (e.g., Johnson and Garrett 2004; Gargett and Garner 2008); results of the random noise testing suggest that the new method is less affected by data fluctuations and therefore identifies a more reliable MLD. Regarding the temperature data of the WOCE section A05, where the depth resolution of the profiles is resampled from 0.04 to $25 \mathrm{dbar}$, the MLDs determined by the relative variance and the differenceinterpolation method are much more consistent with the visually inspected MLD value than the MLDs by other methods. However, the relative variance method derives the most stable MLD and the largest QI among all the methods. These results confirm that the relative variance method is the optimal one for capturing the intersection of a homogeneous ML with an underlying water of sharp gradient, with profiles of any depth resolution.

When applied to the WOCE data of the global ocean, all the methods were found to yield nearly coincident PDFs of $H_{T}$ and $H_{\rho}$, provided that $H_{T}$ or $H_{\rho}$ is larger than 20 dbar. This fact suggests that these methods can produce a fairly reliable MLD for a thick ML. The averaged $H_{T}$ is $6-10$ dbar larger than that of $H_{\rho}$ for the threshold methods, owing to the fact that the compensated layers are much less common than the barrier layers in the global ocean. The relative variance and curvature methods would identify similar $\left\langle H_{T}\right\rangle$ and $\left\langle H_{\rho}\right\rangle$ because they are prone to finding the base of the ML rather than the top of the thermocline from either temperature or density profiles. Therefore, the relative variance method has an advantage in determining the MLD in many cases, especially when the density profile is unavailable, for example, as described in de Boyer Montégut et al. (2004).

Finally, the leading effectiveness of the relative variance method is further confirmed by the fact that the MLD of $94 \%$ of the WOCE temperature profiles can be determined with this method for $\mathrm{QI}>0.5$; this is substantially larger than the $86 \%$ or less corresponding to the MLD derivations of the other methods. A similar conclusion can be drawn based on the density profiles, as listed in Table 2. Furthermore, the MLDs from the relative variance method show similar global seasonal variability as those from other methods but with higher credibility, which is based on the larger QI values. Thus, it has been sufficiently demonstrated that this method has greater potential than other current holding methods in determining accurate MLDs for worldwide temperature and density profiles as well as those from gridded datasets or model outputs.
Acknowledgments. We thank Dr. Brian King for offering the raw data from section A05. We specially thank the anonymous reviewers for their helpful suggestions. This work was supported by the China NSF (41476167, 41406035, and 91752108); the NSF of Guangdong Province, China (2016A030311042); and the Strategic Priority Research Program of the Chinese Academy of Sciences (XDA11030302).

\section{REFERENCES}

Abdulla, C. P., M. A. Alsaafani, T. M. Alraddadi, and A. M. Albarakati, 2016: Estimation of mixed layer depth in the Gulf of Aden: A new approach. PLoS One, 11, e0165136, https:// doi.org/10.1371/journal.pone.0165136.

Brainerd, K., and M. Gregg, 1995: Surface mixed and mixing layer depths. Deep-Sea Res. I, 42, 1521-1543, https://doi.org/ 10.1016/0967-0637(95)00068-H.

Carton, J. A., S. A. Grodsky, and H. Liu, 2008: Variability of the oceanic mixed layer, 1960-2004. J. Climate, 21, 1029-1047, https://doi.org/10.1175/2007JCLI1798.1.

Castro-Morales, K., and J. Kaiser, 2012: Using dissolved oxygen concentrations to determine mixed layer depths in the Bellingshausen Sea. Ocean Sci., 8, 1-10, https://doi.org/10.5194/ os-8-1-2012.

Chu, P. C., and C. Fan, 2010: Optimal linear fitting for objective determination of ocean mixed layer depth from glider profiles. J. Atmos. Oceanic Technol., 27, 1893-1898, https://doi.org/ 10.1175/2010JTECHO804.1.

— mixed layer depth from seaglider data. J. Oceanogr., 67, 219230, https://doi.org/10.1007/s10872-011-0019-2.

de Boyer Montégut, C., G. Madec, A. S. Fischer, A. Lazar, and D. Iudicone, 2004: Mixed layer depth over the global ocean: An examination of profile data and a profile-based climatology. J. Geophys. Res., 109, C12003, https://doi.org/10.1029/ 2004JC002378.

Defant, A., 1936: Die troposphare des atlantischen ozeans. Schichtung und Zierkulation des Atlantischen Ozeans, G. Wiist, Ed., Vol. 6, Part I, Walter de Gruyter, 1925-1929.

Deser, C., M. A. Alexander, and M. S. Timlin, 1996: Upper-ocean thermal variations in the North Pacific during 1970-1991. J. Climate, 9, 1840-1855, https://doi.org/10.1175/1520-0442 (1996)009<1840:UOTVIT >2.0.CO;2.

Dong, S., J. Sprintall, S. T. Gille, and L. Talley, 2008: Southern Ocean mixed-layer depth from Argo float profiles. J. Geophys. Res., 113, C06013, https://doi.org/10.1029/2006JC004051.

Freeland, H., K. Denman, C. Wong, F. Whitney, and R. Jacques, 1997: Evidence of change in the winter mixed layer in the Northeast Pacific Ocean. Deep-Sea Res. I, 44, 2117-2129, https://doi.org/10.1016/S0967-0637(97)00083-6.

Gargett, A., and T. Garner, 2008: Determining Thorpe scales from ship-lowered CTD density profiles. J. Atmos. Oceanic Technol., 25, 1657-1670, https://doi.org/10.1175/ 2008JTECHO541.1.

Godfrey, J., and E. Lindstrom, 1989: The heat budget of the equatorial western Pacific surface mixed layer. J. Geophys. Res., 94, 8007-8017, https://doi.org/10.1029/JC094iC06p08007.

Hanawa, K., and L. D. Talley, 2001: Mode waters. Ocean Circulation and Climate Observing and Modelling the Global Ocean, G. Siedler, J. Church, and J. Gould, Eds., International 
Geophysics Series, Vol. 77, Academic Press, 373-386, https:// doi.org/10.1016/S0074-6142(01)80129-7.

Helber, R. W., A. B. Kara, J. G. Richman, M. R. Carnes, C. N. Barron, H. E. Hurlburt, and T. Boyer, 2012: Temperature versus salinity gradients below the ocean mixed layer. J. Geophys. Res., 117, C05006, https://doi.org/10.1029/ 2011JC007382.

Holte, J., and L. Talley, 2009: A new algorithm for finding mixed layer depths with applications to Argo data and Subantarctic Mode Water formation. J. Atmos. Oceanic Technol., 26, 1920 1939, https://doi.org/10.1175/2009JTECHO543.1.

Johnson, H. L., and C. Garrett, 2004: Effects of noise on Thorpe scales and run lengths. J. Phys. Oceanogr., 34, 2359-2372, https://doi.org/10.1175/jpo2641.1.

Kantha, L., and C. A. Clayson, 1999: Ocean mixed layer. Thermodynamics of Atmospheres and Oceans, J. A. Curry, and P. J. Webster, Eds., International Geophysics Series, Vol. 65, Academic Press, 291-298.

Kara, A. B., P. A. Rochford, and H. E. Hurlburt, 2000: An optimal definition for ocean mixed layer depth. J. Geophys. Res., 105, 16 803-16 821, https://doi.org/10.1029/2000JC900072.

- — - and —, 2003: Mixed layer depth variability over the global ocean. J. Geophys. Res., 108, 3079, https://doi.org/ 10.1029/2000JC000736.

Lavender, K. L., R. E. Davis, and W. B. Owens, 2002: Observations of open-ocean deep convection in the Labrador Sea from subsurface floats. J. Phys. Oceanogr., 32, 511-526, https://doi.org/ 10.1175/1520-0485(2002)032<0511:OOOODC >2.0.CO;2.

Levitus, S., 1982: Climatological Atlas of the World Ocean. NOAA Prof. Paper 13, 173 pp. and 17 microfiche.

Lorbacher, K., D. Dommenget, P. Niiler, and A. Köhl, 2006: Ocean mixed layer depth: A subsurface proxy of ocean-atmosphere variability. J. Geophys. Res., 111, C07010, https://doi.org/ 10.1029/2003JC002157.

Lukas, R., and E. Lindstrom, 1991: The mixed layer of the western equatorial Pacific Ocean. J. Geophys. Res., 96 (Suppl.), 3343 3357, https://doi.org/10.1029/90JC01951.

Neshyba, S., V. T. Neal, and W. Denner, 1971: Temperature and conductivity measurements under Ice Island T-3. J. Geophys. Res., 76, 8107-8120, https://doi.org/10.1029/JC076i033p08107.
Obata, A., J. Ishizaka, and M. Endoh, 1996: Global verification of critical depth theory for phytoplankton bloom with climatological in situ temperature and satellite ocean color data. J. Geophys. Res., 101, 20 657-20 667, https://doi.org/10.1029/ 96JC01734.

Ohno, Y., T. Kobayashi, N. Iwasaka, and T. Suga, 2004: The mixed layer depth in the North Pacific as detected by the Argo floats. Geophys. Res. Lett., 31, L11306, https://doi.org/10.1029/ 2004GL019576.

— N. Iwasaka, F. Kobashi, and Y. Sato, 2009: Mixed layer depth climatology of the North Pacific based on Argo observations. J. Oceanogr., 65, 1-16, https://doi.org/10.1007/s10872-009-0001-4.

Oka, E., and Coauthors, 2015: Decadal variability of Subtropical Mode Water subduction and its impact on biogeochemistry. J. Oceanogr., 71, 389-400, https://doi.org/ 10.1007/s10872-015-0300-x.

Pavlidis, T., and S. L. Horowitz, 1974: Segmentation of plane curves. IEEE Trans. Comput., 23, 860-870, https://doi.org/ 10.1109/T-C.1974.224041.

Price, J. F., R. A. Weller, and R. Pinkel, 1986: Diurnal cycling: Observations and models of the upper ocean response to diurnal heating, cooling and wind mixing. J. Geophys. Res., 91, 8411-8427, https://doi.org/10.1029/JC091iC07p08411.

Sprintall, J., and M. Tomczak, 1992: Evidence of the barrier layer in the surface layer of the tropics. J. Geophys. Res., 97, 73057316, https://doi.org/10.1029/92JC00407.

Thomson, R. E., and I. V. Fine, 2003: Estimating mixed layer depth from oceanic profile data. J. Atmos. Oceanic Technol., 20, 319-329, https:// doi.org/10.1175/1520-0426(2003)020<0319:EMLDFO>2.0.CO;2.

Vallis, G., 2006: Atmospheric and Oceanic Fluid Dynamics: Fundamentals and Large-Scale Circulation. Cambridge University Press, 768 pp., https://doi.org/10.1017/CBO9780511790447.

Wunsch, C., and R. Ferrari, 2004: Vertical mixing, energy, and the general circulation of the ocean. Annu. Rev. Fluid Mech., 36, 281314, https://doi.org/10.1146/annurev.fluid.36.050802.122121.

Wyrtki, K., 1964: The thermal structure of the eastern Pacific Ocean. Dtsch. Hydrogr. Z., 8A, 6-84.

Yu, L., and R. A. Weller, 2007: Objectively analyzed air-sea heat fluxes for the global ice-free oceans (1981-2005). Bull. Amer. Meteor. Soc., 88, 527-539, https://doi.org/10.1175/BAMS-88-4-527. 